\title{
Article \\ Can You Hear the Shape of a Market? Geometric Arbitrage and Spectral Theory
}

\author{
Simone Farinelli ${ }^{1, * \mathbb{C}}$ and Hideyuki Takada ${ }^{2}$ \\ 1 Core Dynamics GmbH, Scheuchzerstrasse 43, CH-8006 Zurich, Switzerland \\ 2 Department of Information Science, Toho University, 2-2-1-Miyama, Funabashi-Shi 274-8510, Chiba, Japan; \\ hideyuki.takada@is.sci.toho-u.ac.jp \\ * Correspondence: simone@coredynamics.ch
}

Citation: Farinelli, S.; Takada, H Can You Hear the Shape of a Market? Geometric Arbitrage and Spectral Theory. Axioms 2021, 10, 242. https:// doi.org/10.3390/axioms10040242

Academic Editor: Hari Mohan Srivastava

Received: 29 August 2021

Accepted: 22 September 2021

Published: 28 September 2021

Publisher's Note: MDPI stays neutral with regard to jurisdictional claims in published maps and institutional affiliations.

Copyright: (C) 2021 by the authors. Licensee MDPI, Basel, Switzerland. This article is an open access article distributed under the terms and conditions of the Creative Commons Attribution (CC BY) license (https:// creativecommons.org/licenses/by/ $4.0 /)$.

\begin{abstract}
Utilizing gauge symmetries, the Geometric Arbitrage Theory reformulates any asset model, allowing for arbitrage by means of a stochastic principal fibre bundle with a connection whose curvature measures the "instantaneous arbitrage capability". The cash flow bundle is the associated vector bundle. The zero eigenspace of its connection Laplacian parameterizes all riskneutral measures equivalent to the statistical one. A market satisfies the No-Free-Lunch-withVanishing-Risk (NFLVR) condition if and only if 0 is in the discrete spectrum of the Laplacian. The Jarrow-Protter-Shimbo theory of asset bubbles and their classification and decomposition extend to markets not satisfying the NFLVR. Euler's characteristic of the asset nominal space and non-vanishing of the homology group of the cash flow bundle are both topological obstructions to NFLVR.
\end{abstract}

Keywords: arbitrage markets; stochastic differential geometry; spectral theory; topological obstructions to arbitrage; asset bubbles

MSC: 91G80; 60G46; 58C40

\section{Introduction}

This paper develops a conceptual structure-called Geometric Arbitrage Theory (GAT) - to link arbitrage modeling in generic markets with spectral theory.

GAT rephrases classical stochastic finance in stochastic differential geometric terms in order to characterize arbitrage. The main idea of the GAT approach consists of modeling markets made of basic financial instruments together with their term structures as principal fibre bundles. The financial features of this market-such as no-arbitrage and equilibrium-are then characterized in terms of standard differential geometric constructions-such as curvature-associated with a natural connection in this fibre bundle. Principal fibre bundle theory has been heavily exploited in theoretical physics as the language in which laws of nature can be best formulated by providing an invariant framework to describe physical systems and their dynamics. These ideas can be carried over to mathematical finance and economics. A market is a financial-economic system that can be described by an appropriate principal fibre bundle. A principle as the invariance of market laws under a change of numéraire can be seen then as gauge invariance. Concepts such as No-Free-Lunch-with-Vanishing-Risk (NFLVR) and No-Unbounded-Profit-withBounded-Risk (NUPBR) have a geometric characterization, which have the Capital Asset Pricing Model (CAPM) as a consequence.

The idea that gauge theories are the natural language for describing economics was first proposed by Malaney and Weinstein in the context of the economic index problem [1,2]. Ilinski [3,4] and Young [5] proposed to view arbitrage as the curvature of a gauge connection, in analogy to some physical theories. Independently, Cliff and Speed [6] further developed Flesaker and Hughston's seminal work [7] and utilized techniques from differential geometry to reduce the complexity of asset models before stochastic modeling. 
In recent years, the research on market models admitting arbitrage has started to receive some well-deserved attention, see $[8,9]$. With this paper, we aim to show that by means of the stochastic differential geometric approach, we can obtain true new results that were not accessible with classical methods.

This paper is structured as follows. Section 2 reviews classical stochastic finance and the Geometric Arbitrage Theory. Arbitrage is seen as the curvature of a principal fibre bundle representing the market which defines the quantity of arbitrage associated with it. Proofs are omitted and can be found in [10] and in [11], where the Geometric Arbitrage Theory has been given a rigorous mathematical foundation utilizing the formal background of stochastic differential geometry as in Elworthy [12], Eméry[13], Hackenbroch and Thalmaier [14], Hsu [15], Schwartz [16] and Stroock [17].

In Section 3 the relationship between arbitrage and spectral theory is investigated. The vector bundle associated with the principal fibre bundle represents the cash flow streams corresponding to the assets and carries a covariant differentiation induced by the connection. The connection Laplacian under the Neumann boundary condition is a self-adjoint operator whose spectrum contains 0 if and only if the market model satisfies the NFLVR condition. If 0 has simple multiplicity, then the market is complete, and vice versa. The eigenspace of the eigenvalue 0 contains all candidates for the Radon-Nikodym derivative of a possible risk-neutral measure with respect to the statistical measure.

The Jarrow-Protter-Shimbo theory of asset bubbles for complete no-arbitrage markets is extended to markets allowing for arbitrage opportunities. The classification of bubbles and their decomposition results are proved for models not necessarily satisfying the NFLVR condition. The connections with Platen-Heath real-world pricing are highlighted.

In Section 4 the cash flow bundle twisted with the exterior algebra of the asset nominal space is a Dirac bundle on which we can apply the Atiyah-Singer index theorem, which takes the form of the Gauss-Bonnet-Chern theorem for manifolds with boundary, from which we can infer a topological obstruction to the NFLVR condition, the nonvanishing of the Euler characteristic of the asset nominal space. By means of the BochnerWeitzenböck formula, we obtain another characterization of NFLVR, namely the presence of 0 in the discrete spectrum of the Dirac Laplacian for the twisted cash flow bundle. Moreover, we obtain another topological obstruction to NFLVR, namely the non-vanishing of the homology group of the cash flow bundle.

Section 5 concludes and Appendix A reviews Nelson's stochastic derivatives.

\section{Geometric Arbitrage Theory Background}

In this section, we explain the main concepts of the Geometric Arbitrage Theory introduced in [10], to which we refer for proofs and examples.

\subsection{The Classical Market Model}

In this subsection, we summarize the classical set up, which will be rephrased in Section 2.4 in differential geometric terms. We basically follow [18] and the ultimate reference [19].

We assume continuous-time trading and that the set of trading dates is $[0,+\infty[$. This assumption is general enough to embed the cases of finite and infinite discrete times as well as one with a finite horizon in continuous time. Note that while it is true that in the real world trading occurs only at discrete times, these are not known a priori and can virtually be any point in the time continuum. This motivates the technical effort of continuous-time stochastic finance.

The uncertainty is modelled by a filtered probability space $(\Omega, \mathcal{A}, \mathbb{P})$, where $\mathbb{P}$ is the statistical (physical) probability measure, $\mathcal{A}=\left\{\mathcal{A}_{t}\right\}_{t \in[0,+\infty}$ an increasing family of sub- $\sigma$ algebras of $\mathcal{A}_{\infty}$, and $\left(\Omega, \mathcal{A}_{\infty}, \mathbb{P}\right)$ is a probability space. The filtration $\mathcal{A}$ is assumed to satisfy the usual conditions, that is:

- $\quad$ right continuity: $\mathcal{A}_{t}=\bigcap_{s>t} \mathcal{A}_{s}$ for all $t \in[0,+\infty[$;

- $\quad \mathcal{A}_{0}$ contains all null sets of $\mathcal{A}_{\infty}$. 
The market consists of finitely many assets indexed by $j=1, \ldots, N$, whose nominal prices are given by the vector valued semimartingale $S:\left[0,+\infty\left[\times \Omega \rightarrow \mathbb{R}^{N}\right.\right.$, denoted by $\left(S_{t}\right)_{t \in[0,+\infty[}$ and adapted to the filtration $\mathcal{A}$. The stochastic process $\left(S_{t}^{j}\right)_{t \in[0,+\infty[}$ describes the price at time $t$ of the $j$ th asset in terms of unit of cash at time $t=0$. More precisely, we assume the existence of a 0 th asset — the cash, a strictly positive semimartingale that evolves according to $S_{t}^{0}=\exp \left(\int_{0}^{t} d u r_{u}^{0}\right)$, where the integrable semimartingale $\left(r_{t}^{0}\right)_{t \in[0,+\infty[}$ represents the continuous interest rate provided by the cash account. One always knows in advance what the interest rate on one's own bank account is, but this can change from time to time. The cash account is therefore considered the locally riskless asset in contrast to the other assets, the risky ones. Subsequently, we will mainly utilize discounted prices, defined as $\hat{S}_{t}^{j}:=S_{t}^{j} / S_{t}^{0}$, representing the asset prices in terms of a current unit of cash.

We remark that there is no need to assume that asset prices are positive. However, there must be at least one strictly positive asset, which in our case is the cash. If we want to renormalize the prices by choosing another asset instead of the cash as reference, i.e., by making it our numéraire, then this asset must have a strictly positive price process. More precisely, a generic numéraire is a portfolio of the original assets $j=0,1,2, \ldots, N$, whose nominal price is represented by a strictly positive stochastic process $\left(B_{t}\right)_{t \in[0,+\infty[}$. The discounted prices of the original assets are then represented in terms of the numéraire by the semimartingales $\hat{S}_{t}^{j}:=S_{t}^{j} / B_{t}$.

We assume that there are no transaction costs and that short sales are allowed. Remark that the absence of transaction costs can be a serious limitation for a realistic model. The filtration $\mathcal{A}$ is not necessarily generated by the price process $\left(S_{t}\right)_{t \in[0,+\infty[}$; sources of information other than prices are allowed. All agents have access to the same information structure, that is, to the filtration $\mathcal{A}$.

Let $v$ be a positive real number. A $v$-admissible strategy $x=\left(x_{t}\right)_{t \in[0,+\infty}$ is a predictable $S$-integrable process for which the Itô integral $\int_{0}^{t} x \cdot d S \geq-v$ a.s. for all $t \geq 0$ with $x_{0}=0$. A strategy is admissible if it is $v$-admissible for some $v \geq 0$.

Definition 1 (Arbitrage). Let the process $\left(S_{t}\right)_{[0,+\infty[}$ be a semimartingale and $\left(x_{t}\right)_{t \in[0,+\infty[}$ be an admissible self-financing strategy. Let us consider trading up to time $T \leq \infty$. The portfolio wealth at time $t$ is given by $V_{t}(x):=V_{0}+\int_{0}^{t} x_{u} \cdot d S_{u}$, and we denote as $K_{0}$ the subset of $L^{0}\left(\Omega, \mathcal{A}_{T}, P\right)$ containing all such $V_{T}(x)$, where $x$ is any admissible self-financing strategy. We define the following:

- $\quad C_{0}:=K_{0}-L_{+}^{0}\left(\Omega, \mathcal{A}_{T}, P\right)$;

- $C:=C_{0} \cap L^{\infty}\left(\Omega, \mathcal{A}_{T}, P\right)$;

- $\quad \bar{C}$ : the closure of $C$ in $L^{\infty}$ with respect to the norm topology;

- $\mathcal{V}^{V_{0}}:=\left\{\left(V_{t}\right)_{t \in[0,+\infty[} \mid V_{t}=V_{t}(x)\right.$, where $x$ is $V_{0}$-admissible $\}$;

- $\mathcal{V}_{T}^{V_{0}}:=\left\{V_{T} \mid\left(V_{t}\right)_{t \in[0,+\infty[} \in \mathcal{V}^{V_{0}}\right\}$;

terminal wealth for $V_{0}$-admissible self-financing strategies.

And let $L_{+}^{\infty}\left(\Omega, \mathcal{A}_{T}, P\right)$ be the set of positive random variables in $L^{\infty}\left(\Omega, \mathcal{A}_{T}, P\right)$. We say that $S$ satisfies:

- $\quad(N A)$ no-arbitrage if and only if $C \cap L_{+}^{\infty}\left(\Omega, \mathcal{A}_{T}, P\right)=\{0\}$;

- (NFLVR) no-free-lunch-with-vanishing-risk if and only if $\bar{C} \cap L_{+}^{\infty}\left(\Omega, \mathcal{A}_{T}, P\right)=\{0\}$;

- (NUPBR) no-unbounded-profit-with-bounded-risk if and only if $\mathcal{V}_{T}^{V_{0}}$ is bounded in $L^{0}$ for some $V_{0}>0$.

The relationship between these three different types of arbitrage has been elucidated in $[20,21]$, with the proof of the following result.

Theorem 1.

$$
(N F L V R) \Leftrightarrow(N A)+(N U P B R) .
$$


Theorem 2 (First fundamental theorem of asset pricing). The market $(S, \mathcal{A})$ satisfies the NFLVR condition if and only if there exists an equivalent local martingale measure $P^{*}$.

Remark 1. In the first fundamental theorem of asset pricing, we just assumed that the price process $S$ is locally bounded. If $S$ is bounded, then NFLVR is equivalent to the existence of a martingale measure. But without this additional assumption, NFLVR only implies the existence of a local martingale measure, i.e., a local martingale which is not a martingale. This distinction is important because the difference between a security price process being a strict local martingale versus a martingale under a probability $P^{*}$ is related to the existence of asset price bubbles.

Definition 2 (Complete market). The market $(S, \mathcal{A})$ is complete on $[0, T]$ if for all contingent claims $C \in L_{+}\left(P^{*}, \mathcal{A}_{T}\right):=\left\{C: \Omega \rightarrow\left[0,+\infty\left[\mid C\right.\right.\right.$ is $\mathcal{A}_{T}-$ measurable, and $\left.\mathbb{E}_{0}^{P^{*}}[|C|]<+\infty\right\}$ there exists an admissible self-financing strategy $x$ such that $C=V_{T}(x)$.

Theorem 3 (Second fundamental theorem of asset pricing). Given that $(S, \mathcal{A})$ satisfies the NFLVR condition, the market is complete on $[0, T]$ if and only if the equivalent local martingale $P^{*}$ is unique.

Definition 3 (Dominance). The $j$-th security $S^{j}=\left(S_{t}^{j}\right)_{t \in[0 . T]}$ is undominated on $[0, T]$ if there is no admissible strategy $\left(x_{t}\right)_{t \in[0, T]}$ such that:

$$
S_{0}^{j}+(x \cdot S)_{T} \geq S_{T}^{j} \quad \text { a.s. and } \quad P\left[S_{0}^{j}+(x \cdot S)_{T}>S_{T}^{j}\right]>0 .
$$

We say that $S$ satisfies the no dominance condition (ND) on $[0, T]$ if and only if each $S^{j}$, $j=0,1, \ldots, N$ is undominated on $[0, T]$.

Definition 4 (Economy). An economy consists of a market given by $(S, \mathcal{A})$ and a finite number of investors $k=1, \ldots, K$ characterized by their beliefs, information, preferences, and endowment. Moreover, there is a single consumption good that is perishable. The price of the consumption good in units of the cash account is denoted as $\Psi=\left(\Psi_{t}\right)_{t \in[0, T]}$. We assume that $\Psi$ is strictly positive. The $k$-th investor is characterized by the following quantities:

- Beliefs and information: $\left(P_{k}, \mathcal{A}\right)$. We assume that the investor's beliefs $P_{k}$ are equivalent to $P$. All investors have the same information filtration $\mathcal{A}$.

- Utility function: $U_{k}:[0, T] \times[0,+\infty[\rightarrow \mathbb{R}$ and $\mu$ is a probability measure on $[0, T]$ with $\mu(\{T\})>0$ such that for every $t$ in the support of $\mu$, the function $U_{k}(t, \cdot)$ is strictly increasing. We also assume $\lim _{v \rightarrow+\infty} U_{k}(T, v)=+\infty$. The utility that agent $k$ derives from consuming $c_{t} \mu(d t)$ at each time $t \leq T$ is as follows:

$$
\mathcal{U}_{k}(c)=\mathbb{E}_{0}^{k}\left[\int_{0}^{T} U_{k}\left(t, c_{t}\right) \mu(d t)\right],
$$

where $\mathbb{E}^{k}$ is the expectation with respect to $P_{k}$. Since $\mu(\{T\})>0$, the utility is strictly increasing in the final consumption $c_{T}$.

- Initial wealth: $v_{k}$. Given a trading strategy $x=\left(x^{1}, \cdots, x^{N}\right)$, the investor will be required to choose his initial holding $x_{0}^{0}$ in the cash account such that:

$$
v_{k}=x_{0}^{0}+\sum_{j=1}^{N} x_{0}^{j} S_{0}^{i}
$$

- Stochastic endowment stream: $\epsilon_{t}^{k}, t<T$ of the commodity. This means that the investors receive $\epsilon_{t}^{k} \mu(d t)$ units of the commodity at time $t \leq T$. The cumulative endowment of the $k$-th investor in units of the cash account is given by the following equation:

$$
\mathcal{E}_{t}^{k}:=\int_{0}^{t} \Psi_{s} \epsilon_{s}^{k} \mu(d s)
$$


Definition 5 (Consumption plan and strategy). A pair $\left(c_{t}^{k}, x_{t}^{k}\right)_{t \in[0, T]}$ is called admissible if $\left(c_{t}^{k}\right)_{t \in[0, T]}$ is progressively measurable with respect to the filtration $\mathcal{A},\left(x_{t}^{k}\right)_{t \in[0, T]}$ is admissible in the usual sense, and it generates a wealth process $V^{k}=\left(V_{t}^{K}\right)_{t \in[0, T]}$ with non-negative terminal wealth $V_{T}^{k} \geq 0$.

Definition 6 (Equilibrium). Given an economy $\left(\left\{P_{k}\right\}_{k=1, \ldots, K}\left(\mathcal{A}_{t}\right)_{t \in[0, T]}\right.$, $\left.\left\{\epsilon_{k}\right\}_{k=1, \ldots, K},\left\{U_{k}\right\}_{k=1, \ldots, K}\right)$, a consumption good price index $\Psi$, financial assets $S=\left[S^{0}, S^{1}, \ldots, S^{N}\right]^{\dagger}$, and investor consumption-investment plans $\left(\hat{c}^{k}, \hat{x}^{k}\right)$ for $k=1, \ldots K$, the pair $(\Psi, S)$ is an equilibrium price process if for all $t \leq T P$-a.s. the following conditions are satisfied:

- Securities markets clear:

$$
\sum_{k=1}^{K} \hat{x}_{t}^{k, j}=\alpha^{j} \quad(j=0,1, \ldots, N),
$$

where $\alpha^{j}$ is the aggregate net supply of the $j$-th security. It is assumed that each $\alpha^{j}$ is nonrandom and constant over time, with $\alpha^{0}=0$ and $\alpha^{j}>0$ for $j=1, \ldots, N$.

- Commodity markets clear:

$$
\sum_{k=1}^{K} \hat{c}_{t}^{k}=\sum_{k=1}^{K} \epsilon_{t}^{k}
$$

- Investors' choices are optimal: $\left(\hat{c}^{k}, \hat{x}^{k}\right)$ solves the $k$-th investor's utility maximization problem

$$
u_{k}(x):=\sup \left\{U_{k}(c) \mid c \text { admissible consumption plan, } x^{k}=x\right\},
$$

and the optimal value is finite.

Definition 7 (Efficiency). A market model given by $S$ is called efficient on $[0, T]$ with respect to $\left(\mathcal{A}_{t}\right)_{t \in[0, T]}$, i.e., $(E)$, if there exists a consumption good price index $\Psi$ and an economy $\left(\left\{P_{k}\right\}_{k=1, \ldots, K},\left(\mathcal{A}_{t}\right)_{t \in[0, T]},\left\{\epsilon_{k}\right\}_{k=1, \ldots, K},\left\{U_{k}\right\}_{k=1, \ldots, K}\right)$, for which $(\Psi, S)$ is an equilibrium price process on $[0, T]$.

In $[22,23]$ we find the proof of the following result.

Theorem 4 (Third fundamental theorem of asset pricing, characterization of efficiency). Let $(S, \mathcal{A})$ be a market. The following statements are equivalent:

(i) $(E):(S, \mathcal{A})$ is efficient in $[0, T]$;

(ii) $(S, \mathcal{A})$ satisfies both (NFLVR) and (ND) on $[0, T]$;

(iii) (EMM): There exists a probability $P^{*}$ equivalent to $P$ such that $S$ is a $\left(P^{*}, \mathcal{A}\right)$ martingale on $[0, T]$.

\subsection{Geometric Reformulation of the Market Model: Primitives}

We are going to introduce a more general representation of the market model introduced in Section 2.1, which better suits the arbitrage modeling task.

Definition 8. A gauge is an ordered pair of two $\mathcal{A}$-adapted real valued semimartingales $(D, P)$, where $D=\left(D_{t}\right)_{t \geq 0}:\left[0,+\infty\left[\times \Omega \rightarrow \mathbb{R}\right.\right.$ is called a deflator and $P=\left(P_{t, s}\right)_{t, s}: \mathcal{T} \times \Omega \rightarrow \mathbb{R}$, which is called a term structure, is considered a stochastic process with respect to the time $t$, termed valuation date, and $\mathcal{T}:=\left\{(t, s) \in\left[0,+\infty\left[{ }^{2} \mid s \geq t\right\}\right.\right.$. The parameter $s \geq t$ is referred to as maturity date. The following properties must be satisfied a.s. for all $t, s$ such that $s \geq t \geq 0$ :

(i) $P_{t, s}>0$;

(ii) $P_{t, t}=1$.

Remark 2. Deflators and term structures can be considered outside the context of fixed income. An arbitrary financial instrument is mapped to a gauge $(D, P)$ with the following economic interpretation: 
- Deflator: $D_{t}$ is the value of the financial instrument at time texpressed in terms of some numéraire. If we choose the cash account, the 0-th asset, as numéraire, then we can set $D_{t}^{j}:=\hat{S}_{t}^{j}=\frac{S_{t}^{j}}{S_{t}^{0}} \quad(j=1, \ldots N)$.

- $\quad$ Term structure: $P_{t, s}$ is the value at time $t$ (expressed in units of deflator at time $t$ ) of a synthetic zero coupon bond with maturity s delivering one unit of financial instrument at time s. It represents a term structure of forward prices with respect to the chosen numéraire.

We point out that there is no unique choice for deflators and term structures describing an asset model. For example, if a set of deflators qualifies, then we can multiply every deflator by the same positive semimartingale to obtain another suitable set of deflators. Of course, term structures have to be modified accordingly. The term "deflator" is clearly inspired by actuarial mathematics. In the present context, it refers to a nominal asset value division by a strictly positive semimartingale (which can be the state price deflator, if this exists, and it is made the numéraire). There is no need to assume that a deflator is a positive process. However, if we want to make an asset our numéraire, then we have to make sure that the corresponding deflator is a strictly positive stochastic process.

\subsection{Geometric Reformulation of the Market Model: Portfolios}

We would now like to introduce transforms of deflators and term structures in order to group gauges containing the same (or less) stochastic information. In this regard, we will consider deterministic linear combinations of assets modelled by the same gauge (e.g., zero bonds of the same credit quality with different maturities).

Definition 9. Let $\pi:[0,+\infty[\longrightarrow \mathbb{R}$ be a deterministic cash flow intensity (possibly generalized) function. It induces a gauge transform $(D, P) \mapsto \pi(D, P):=(D, P)^{\pi}:=\left(D^{\pi}, P^{\pi}\right)$ by the following formula:

$$
D_{t}^{\pi}:=D_{t} \int_{0}^{+\infty} d h \pi_{h} P_{t, t+h} \quad P_{t, s}^{\pi}:=\frac{\int_{0}^{+\infty} d h \pi_{h} P_{t, s+h}}{\int_{0}^{+\infty} d h \pi_{h} P_{t, t+h}} .
$$

Proposition 1. Gauge transforms induced by cash flow vectors have the following property:

$$
\left((D, P)^{\pi}\right)^{v}=\left((D, P)^{v}\right)^{\pi}=(D, P)^{\pi * v}
$$

where $*$ denotes the convolution product of two cash flow vectors or intensities respectively:

$$
(\pi * v)_{t}:=\int_{0}^{t} d h \pi_{h} v_{t-h}
$$

The convolution of two non-invertible gauge transforms is non-invertible. The convolution of a non-invertible with an invertible gauge transform is non-invertible.

Definition 10. If the term structure is differentiable with respect to the maturity date, it can be written as a functional of the instantaneous forward rate fdefined as follows:

$$
f_{t, s}:=-\frac{\partial}{\partial s} \log P_{t, s}, \quad P_{t, s}=\exp \left(-\int_{t}^{s} d h f_{t, h}\right),
$$

and

$$
r_{t}:=\lim _{s \rightarrow t^{+}} f_{t, s}
$$

is termed short rate.

Remark 3. The special choice of vanishing interest rate $r \equiv 0$ or flat term structure $P \equiv 1$ for all assets corresponds to the classical model, where only asset prices and their dynamics are relevant. 


\subsection{Arbitrage Theory in a Differential Geometric Framework}

Now we are in the position to rephrase the asset model presented in Section 2.1 in terms of a natural geometric language. Given $N$ base assets, we want to construct a portfolio theory and study arbitrage, and thus we cannot a priori assume the existence of a risk-neutral measure or a state price deflator. In terms of differential geometry, we will adopt the mathematician's and not the physicist's approach. The market model is seen as a principal fibre bundle of the (deflator, term structure) pairs, discounting and foreign exchange as a parallel transport, numéraire as the global section of the gauge bundle, and arbitrage as curvature. The no-free-lunch-with-vanishing-risk condition is proved to be equivalent to a zero-curvature condition.

\subsubsection{Market Model as Principal Fibre Bundle}

Let us consider, in continuous time, a market with $N$ assets and a numéraire. A general portfolio at time $t$ is described by the vector of nominals $x \in \mathfrak{X}$, for an open set $\mathfrak{X} \subset \mathbb{R}^{N}$. By nominals $x^{1}, \ldots, x^{N}$ we mean the number of assets that we hold in our portfolio. Following Definition 8 , the asset model consisting in $N$ synthetic zero bonds is described by means of the following gauges:

$$
\left(D^{j}, P^{j}\right)=\left(\left(D_{t}^{j}\right)_{t \in[0,+\infty[}\left(P_{t, s}^{j}\right)_{s \geq t}\right),
$$

where $D^{j}$ denotes the deflator and $P^{j}$ the term structure. This can be written as follows:

$$
P_{t, s}^{j}=\exp \left(-\int_{t}^{s} f_{t, u}^{j} d u\right)
$$

where $f^{j}$ is the instantaneous forward rate process for the $j$-th asset and the corresponding short rate is given by $r_{t}^{j}:=\lim _{u \rightarrow 0^{+}} f_{t, u}^{j}$. For a portfolio with nominals $x \in \mathfrak{X} \subset \mathbb{R}^{N}$, we define:

$$
D_{t}^{x}:=\sum_{j=1}^{N} x_{j} D_{t}^{j} \quad f_{t, u}^{x}:=\sum_{j=1}^{N} \frac{x_{j} D_{t}^{j}}{\sum_{j=1}^{N} x_{j} D_{t}^{j}} f_{t, u}^{j} \quad P_{t, s}^{x}:=\exp \left(-\int_{t}^{s} f_{t, u}^{x} d u\right) .
$$

The short rate writes as follows:

$$
r_{t}^{x}:=\lim _{u \rightarrow 0^{+}} f_{t, u}^{x}=\sum_{j=1}^{N} \frac{x_{j} D_{t}^{j}}{\sum_{j=1}^{N} x_{j} D_{t}^{j}} r_{t}^{j} .
$$

The image space of all possible strategies reads as follows:

$$
M:=\{(t, x) \in[0,+\infty[\times \mathfrak{X}\} .
$$

In Section 2.3, cash flow intensities and the corresponding gauge transforms were introduced. They have the structure of an Abelian semigroup:

$$
H:=\mathcal{E}^{\prime}\left(\left[0,+\infty[, \mathbb{R})=\left\{F \in \mathcal{D}^{\prime}([0,+\infty[) \mid \operatorname{supp}(F) \subset[0,+\infty[\text { is compact }\},\right.\right.\right.
$$

where the semigroup operation on distributions with compact support is the convolution (see [24], Chapter IV), which extends the convolution of regular functions as defined by Formula (11).

Definition 11. The Market Fibre Bundle is defined as the fibre bundle of the following gauges:

$$
\mathcal{B}:=\left\{\left(D_{t}^{x}, P_{t,}^{x} .\right)^{\pi} \mid(t, x) \in M, \pi \in G\right\} .
$$


The cash flow intensities defining invertible transforms constitute an Abelian group:

$$
G:=\{\pi \in H \mid \text { it exists } v \in H \text { such that } \pi * v=\delta\} \subset \mathcal{E}^{\prime}([0,+\infty[, \mathbb{R}) .
$$

From Proposition 1, we obtain the following theorem.

Theorem 5. The market fibre bundle $\mathcal{B}$ has the structure of a G-principal fibre bundle given by the action:

$$
\begin{aligned}
\mathcal{B} \times G & \longrightarrow \mathcal{B} \\
((D, P), \pi) & \mapsto(D, P)^{\pi}=\left(D^{\pi}, P^{\pi}\right)
\end{aligned}
$$

The group $G$ acts freely and differentiably on $\mathcal{B}$ to the right.

\subsubsection{Nelson $\mathcal{D}$ Weak Differentiable Market Model}

We continue to reformulate the classic asset model introduced in Section 2.1 in terms of stochastic differential geometry.

Definition 12. A Nelson $\mathcal{D}$ weak differentiable market model for $N$ assets is described by $N$ gauges, which are Nelson $\mathcal{D}$ weak differentiable with respect to the time variable. More exactly, for all $t \in[0,+\infty[$ and $s \geq t$, there is an open time interval $I \ni t$ such that for the deflators $D_{t}:=\left[D_{t}^{1}, \ldots, D_{t}^{N}\right]^{\dagger}$ and the term structures $P_{t, s}:=\left[P_{t, s}^{1}, \ldots, P_{t, s}^{N}\right]^{\dagger}$, the latter seen as processes in $t$ and parameter $s$, there exists a $\mathcal{D}$ weak $t$-derivative (see Appendix $A$ ). The short rates are defined by $r_{t}:=\lim _{s \rightarrow t^{-}} \frac{\partial}{\partial s} \log P_{t s}$.

A strategy is a curve $\gamma: I \rightarrow X$ in the portfolio space parameterized by the time. This means that the allocation at time $t$ is given by the vector of nominals $x_{t}:=\gamma(t)$. We denote as $\bar{\gamma}$ the lift of $\gamma$ to $M$, that is $\bar{\gamma}(t):=(\gamma(t), t)$. A strategy is said to be closed if it represented by a closed curve. $A$ weak $\mathcal{D}$-admissible strategy is predictable and $\mathcal{D}$-weak differentiable.

Remark 4. We require weak $\mathcal{D}$-differentiability and not strong $\mathcal{D}$-differentiability because imposing a priori regularity properties on the trading strategies corresponds to restricting the class of admissible strategies with respect to the classical notion of Delbaen and Schachermayer. Every (no-)arbitrage consideration depends crucially on the chosen definition of admissibility. Therefore, restricting the class of admissible strategies may lead to the automatic exclusion of potential arbitrage opportunities, leading to vacuous statements of FTAP-like results. An admissibile strategy in the classic sense (see Section 2) is weak $\mathcal{D}$-differentiable.

In general the allocation can depend on the state of the nature, i.e., $x_{t}=x_{t}(\omega)$ for $\omega \in \Omega$.

Proposition 2. A weak $\mathcal{D}$-admissible strategy is self-financing if and only if:

$$
\mathcal{D}\left(x_{t} \cdot D_{t}\right)=x_{t} \cdot \mathcal{D} D_{t}-\frac{1}{2} \mathfrak{D}_{*}\langle x, D\rangle_{t} \text { or } \mathcal{D} x_{t} \cdot D_{t}=-\frac{1}{2} \mathfrak{D}_{*}\langle x, D\rangle_{t} \text { or } \mathfrak{D} x_{t} \cdot D_{t}=0,
$$

almost surely. The bracket $\langle\cdot, \cdot\rangle$ denotes the continuous part of the quadratic covariation.

For the remainder of this paper, unless otherwise stated, we will only deal with weak $\mathcal{D}$ differentiable market models, weak $\mathcal{D}$ differentiable strategies, and, when necessary, with weak $\mathcal{D}$ differentiable state price deflators. All Itô processes are weak $\mathcal{D}$ differentiable, so that the class of considered admissible strategies is very large.

\subsubsection{Arbitrage as Curvature}

The Lie algebra of $G$ is the function space of all real valued functions on $[0,+\infty[$ denoted as:

$$
\mathfrak{g}=\mathbb{R}^{[0,+\infty[}
$$


and therefore commutative. Following Ilinski's idea [4], we motivate the choice of a particular $\mathfrak{g}$-valued connection 1-form by the fact that it allows us to encode portfolio rebalancing (or foreign exchange) and discounting as parallel transport.

Theorem 6. With the choice of connection

$$
\chi(x, t, g) .(\delta x, \delta t):=\left(\frac{D_{t}^{\delta x}}{D_{t}^{x}}-r_{t}^{x} \delta t\right) g,
$$

the stochastic parallel transport in $\mathcal{B}$ has the following financial interpretations:

- Parallel transport along the nominal directions ( $x$-lines) corresponds to a multiplication by an exchange rate;

- $\quad$ Parallel transport along the time direction (t-line) corresponds to a division by a stochastic discount factor.

Proof. We refer to Theorem 28 in [10].

Recall that the time derivatives needed to define the parallel transport along the time lines have to be understood in Stratonovich's sense. We see that the bundle is trivial, because it has a global trivialization, but the connection is not trivial. The connection $\chi$ writes as a linear combination of basis differential forms:

$$
\chi(x, t, g)=\left(\frac{1}{D_{t}^{x}} \sum_{j=1}^{N} D_{t}^{j} d x_{j}-r_{t}^{x} d t\right) g .
$$

The $g$-valued curvature 2-form is defined as follows:

$$
R:=d \chi+[\chi, \chi]
$$

which means that by this, for all $(x, t, g) \in \mathcal{B}$ and for all $\xi, \eta \in T_{(x, t)} M$,

$$
R(x, t, g)(\xi, \eta):=d \chi(x, t, g)(\xi, \eta)+[\chi(x, t, g)(\xi), \chi(x, t, g)(\eta)] .
$$

Note that the Lie algebra being commutative, the Lie bracket $[\cdot, \cdot]$ vanishes. After some calculations, we obtain the following:

$$
R(x, t, g)=\frac{g}{D_{t}^{x}} \sum_{j=1}^{N} D_{t}^{j}\left(r_{t}^{x}+\mathcal{D} \log \left(D_{t}^{x}\right)-r_{t}^{j}-\mathcal{D} \log \left(D_{t}^{j}\right)\right) d x_{j} \wedge d t,
$$

summarized as the following proposition.

Proposition 3 (Curvature Formula). Let $R$ be the curvature. Then, the following quality holds:

$$
R(t, x, g)=g d t \wedge d_{x}\left[\mathcal{D} \log \left(D_{t}^{x}\right)+r_{t}^{x}\right]
$$

The following result characterizes arbitrage as curvature.

Theorem 7 (No-Arbitrage). The following assertions are equivalent:

(i) The market model (with base assets and futures with discounted prices D and P) satiffies the no-free-lunch-with-vanishing-risk condition;

(ii) There exists a positive martingale $\beta=\left(\beta_{t}\right)_{t \geq 0}$ such that deflators and short rates satisfy, for all portfolio nominals and all times, the condition

$$
r_{t}^{x}=-\mathcal{D} \log \left(\beta_{t} D_{t}^{x}\right)
$$


(iii) There exists a positive martingale $\beta=\left(\beta_{t}\right)_{t \geq 0}$ such that deflators and term structures satisfy, for all portfolio nominals and all times, the condition

$$
P_{t, s}^{x}=\frac{\mathbb{E}_{t}\left[\beta_{s} D_{s}^{x}\right]}{\beta_{t} D_{t}^{x}} .
$$

This motivates the following definition.

Definition 13. The market model satisfies the zero curvature (ZC) if and only if the curvature vanishes a.s.

Therefore, we have following implications relying on two different definitions of no-arbitrage:

\section{Corollary 1.}

$$
(N F L V R) \Rightarrow(Z C)
$$

As proved in [11], the two weaker notions of arbitrage-the zero curvature and the no-unbounded-profit-with-bounded-risk-are satisfied.

\section{Theorem 8 .}

$$
(N U P B R) \Rightarrow(Z C)
$$

The converse is true for special cases of Itô's dynamics for asset values and term structures (see [11]).

Remark 5. Let us consider some special cases of Theorem 7.

1. The components of $r$ are equal: For example, in the classical model, where there are no term structures (i.e., $r \equiv 0$ ),

(a) D and $r$ are constant over time: NFLVR is satisfied;

(b) D and $r$ are deterministic and not constant over time: NFLVR is never satisfied.

2. The components of $r$ are not equal:

(a) D and $r$ are constant over time: NFLVR is never satisfied;

(b) D and $r$ are deterministic and not constant over time: NFLVR can be satisfied if (ii) or (iii) hold true.

2.4.4. Expected Utility Maximization and the CAPM Formula

Definition 14 (EUM). The expected utility maximization problem for the final wealth over the period $[0, t]$ for a given utility function $u$ reads as follows:

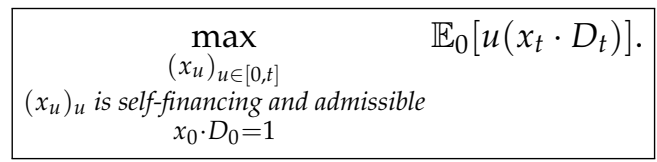

We denote as (EUM) the existence of a solution and its uniqueness for (35).

As proved in [11], e.g., the existence of a solution for an expected utility maximization problem and the no-unbounded-profit-with-bounded-risk is satisfied.

\section{Theorem 9.}

$$
(N U P B R) \Rightarrow(E U M)
$$

Asset returns and the market portfolio return are related at the expected value level by means of sensitivities. The relationship is the following equilibrium result. 
Theorem 10 (CAPM). Let

$$
R_{[0, t]}:=\frac{D_{t}}{D_{0}}-1 \quad R_{[0, t]}^{M}:=\frac{D_{t}^{x_{t}^{M}}}{D_{0}^{x_{0}^{M}}}-1
$$

be the discounted assets total returns and the discounted market portfolio total return. If we assume that the expected utility of the final wealth of every portfolio is maximized, then

$$
\mathbb{E}_{0}\left[R_{[0, t]}\right]=\frac{\operatorname{Cov}_{0}\left(R_{[0, t]}, R_{[0, t]}^{M}\right)}{\operatorname{Var}_{0}\left(R_{[0, t]}^{M}\right)} \mathbb{E}_{0}\left[R_{[0, t]}^{M}\right.
$$

Proof. If we choose a quadratic utility function

$$
u(v):=v-\frac{\lambda}{2} v^{2}
$$

where $\lambda$ denotes the risk aversion parameter, and we assume that only at times $\{0, t\}$ rebalancing during the interval $[0, t]$ is allowed, then the expected utility maximization problem becomes

$$
\max _{\substack{x_{0} \\ x_{0} \cdot D_{0}=1}} \mathbb{E}_{0}\left[\left(x_{t} \cdot D_{t}\right)-\frac{\lambda}{2}\left(x_{t} \cdot D_{t}\right)^{2}\right] \text {, }
$$

which is equivalent to

$$
\max _{\substack{x_{0} \\ x_{0} \cdot D_{0}=1}} \mathbb{E}_{0}\left[\left(x_{t} \cdot D_{t}\right)-\frac{\lambda}{2}\left(\left(x_{t} \cdot D_{t}\right)^{2}-\mathbb{E}_{0}\left[x_{t} \cdot D_{t}\right]\right)\right],
$$

and to

$$
\max _{\substack{w_{0} \\ w_{0} \cdot e=1}} w_{0}^{\dagger} \mathbb{E}_{0}\left[R_{[0, t]}\right]-\frac{\lambda}{2} w_{0}^{\dagger} \operatorname{VCM}_{0}\left(R_{[0, t]}\right) w_{0}
$$

where $w_{0}:=\frac{x_{0} D_{0}}{x_{0} \cdot D_{0}}$ are the portfolio weights. The solution is the market portfolio

$$
w_{0}^{M}=\frac{\hat{w}_{0}^{M}}{\sqrt{\left(\hat{w}_{0}^{M}\right)^{\dagger} \hat{w}_{0}^{M}}}, \text { where } \quad \hat{w}_{0}^{M}:=\frac{1}{\lambda} \operatorname{VCM}_{0}\left(R_{[0, t]}\right)^{-1} \mathbb{E}_{0}\left[R_{[0, t]}\right]
$$

Therefore, we have by (43)

$$
\begin{aligned}
\mathbb{E}_{0}\left[R_{[0, t]}\right] & =\lambda \operatorname{VCM}_{0}\left(R_{[0, t]}\right) \hat{w}_{0}^{M}=\frac{\lambda}{\left|\hat{w}_{0}^{M}\right|} \operatorname{VCM}_{0}\left(R_{[0, t]}\right) w_{0}^{M}= \\
& =\frac{\lambda}{\left|\hat{w}_{0}^{M}\right|} \operatorname{Cov}_{0}\left(R_{[0, t]}, R_{[0, t]}^{M}\right),
\end{aligned}
$$

and by multiplication with $w_{0}^{M}$

$$
\mathbb{E}_{0}\left[R_{[0, t]}^{M}\right]=\frac{\lambda}{\left|\hat{w}_{0}^{M}\right|^{2}}\left(w_{0}^{M}\right)^{\dagger} \operatorname{VCM}_{0}\left(R_{[0, t]}\right) w_{0}^{M}=\frac{\lambda}{\left|\hat{w}_{0}^{M}\right|^{2}} \operatorname{Var}_{0}\left(R_{[0, t]}^{M}\right),
$$

from which we infer that

$$
\frac{\lambda}{\left|\hat{w}_{0}^{M}\right|^{2}}=\frac{\mathbb{E}_{0}\left[R_{[0, t]}^{M}\right]}{\operatorname{Var}_{0}\left(R_{[0, t]}^{M}\right)}
$$


which, inserted into (45), leads to

$$
\mathbb{E}_{0}\left[R_{[0, t]}\right]=\frac{\operatorname{Cov}_{0}\left(R_{[0, t]}, R_{[0, t]}^{M}\right)}{\operatorname{Var}_{0}\left(R_{[0, t]}^{M}\right)} \mathbb{E}_{0}\left[R_{[0, t]}\right]
$$

and the proof is completed.

Remark 6. The vector

$$
\beta_{0}:=\frac{\operatorname{Cov}_{0}\left(R_{[0, t]}, R_{[0, t]}^{M}\right)}{\operatorname{Var}_{0}\left(R_{[0, t]}^{M}\right)}
$$

contains the sensitivities of the expected asset returns with respect to the expected market portfolio return. We can compute the CAPM in its classical form for the returns of the asset as follows:

$$
r_{[0, t]}:=\frac{S_{t}}{S_{0}}-1=\left(1+R_{[0, t]}\right) \exp \left(+\int_{0}^{t} d u r_{u}^{0}\right)-1
$$

obtaining

$$
\mathbb{E}_{0}\left[r_{[0, t]}\right]-r_{[0, t]}^{f}=\frac{\operatorname{Cov}_{0}\left(r_{[0, t]}, r_{[0, t]}^{M}\right)}{\operatorname{Var}_{0}\left(r_{[0, t]}^{M}\right)}\left(\mathbb{E}_{0}\left[r_{[0, t]}\right]-r_{[0, t]}^{f}\right),
$$

where $r_{[0, t]}^{f}:=\exp \left(+\int_{0}^{t} d u r_{u}^{0}\right)-1$ is the risk-free return.

Remark 7. The different arbitrage concepts and the Capital Asset Pricing Model are related in the following logical representation:

$$
(E M M) \Leftrightarrow(E) \Leftrightarrow\left\{\begin{array}{l}
(N F L V R) \Leftrightarrow\left\{\begin{array}{l}
(N U P B R) \Rightarrow\left\{\begin{array}{l}
(E U M) \\
(\mathrm{ZC}) \\
(N A)
\end{array}\right. \\
(N D)
\end{array}\right\} \Rightarrow(C A P M)
\end{array}\right.
$$

\section{Spectral Theory}

3.1. Cash Flows as Sections of the Associated Vector Bundle

Definition 15 (Cash Flow Bundle). By choosing the fiber $V:=\mathbb{R}^{[0,+\infty[}$ and the representation $\rho: G \rightarrow G L(V)$ induced by the gauge transform definition, and therefore satisfying the homomorphism relation $\rho\left(g_{1} * g_{2}\right)=\rho\left(g_{1}\right) \rho\left(g_{2}\right)$, we obtain the associated vector bundle $\mathcal{V}$. Its sections represent cash flow streams - expressed in terms of the deflators-generated by portfolios of the base assets. If $v=\left(v_{t}^{x}\right)_{(t, x) \in M}$ is the deterministic cash flow stream, then its value at time $t$ is equal to:

- $\quad$ the deterministic quantity $v_{t}^{x}$, if the value is measured in terms of the deflator $D_{t}^{x}$;

- the stochastic quantity $v_{t}^{x} D_{t}^{x}$, if the value is measured in terms of the numéraire (e.g., the cash account for the choice $D_{t}^{j}:=\hat{S}_{t}^{j}$ for all $\left.j=1, \ldots, N\right)$.

The bundle $\mathcal{V}$ over the time-nominals-space $M=[0, T] \times \mathfrak{X}$ is called the Cash Flow Bundle.

In the general theory of principal fibre bundles, gauge transforms are bundle automorphisms preserving the group action and equal to the identity on the base space. Gauge transforms of $\mathcal{B}$ are naturally isomorphic to the sections of the bundle $\mathcal{B}$ (See Theorem 3.2.2 in [25]). Since $G$ is Abelian, right multiplications are gauge transforms. Hence, there is a bijective correspondence between gauge transforms and cash flow intensities admitting an inverse. This justifies the terminology introduced in Definition 9. 


\subsection{The Connection Laplacian associated with the Market Model}

The connection $\chi$ on the market principal fibre bundle $\mathcal{B}$ defined in Theorem 6 induces a covariant differentiation $\nabla^{\mathcal{V}}$ on the associated vector bundle $\mathcal{V}$, with the same interpretation for the corresponding parallel transport as that in Theorem 6 for the principal fibre bundle, i.e., portfolio rebalancing along the asset nominal dimensions and discounting along the time dimension. More exactly, we have the following proposition.

Proposition 4. Let us extend the coordinate vector $x \in \mathbb{R}^{N}$ with a 0 th component given by the time t. Let $X=\sum_{j=0}^{N} X_{j} \frac{\partial}{\partial x_{j}}$ be a vector field over $M$ and $f=\left(f_{s}\right)_{s}$ a section of the cash flow bundle $\mathcal{V}$. Then

$$
\nabla_{X}^{\mathcal{V}} f_{t}=\sum_{j=0}^{N}\left(\frac{\partial f_{t}}{\partial x_{j}}+K_{j} f_{t}\right) X_{j}
$$

where

$$
\begin{aligned}
& K_{0}(x)=-r_{t}^{x} \\
& K_{j}(x)=\frac{D_{t}^{j}}{D_{t}^{x}} \quad(1 \leq j \leq N) .
\end{aligned}
$$

Proof. The construction of a covariant differentiation on the associated vector bundle starting from a connection on a principal fibre bundle is a generic procedure in differential geometry. The connection $\chi$ is a Lie algebra $\mathfrak{g}=\mathbb{R}^{[0,+\infty[}$ valued 1 -form on $\mathcal{B}$, and we can decompose the connection as $\chi(x, g)=g K(x)$, where $K(x):=\sum_{j=0}^{N} K_{j}(x) d x_{j}$. The differential map $T_{e} \rho: \mathfrak{g} \rightarrow \mathcal{L}\left(\mathbb{R}^{[0,+\infty}[)\right.$ of the representation $\rho: G \rightarrow \mathrm{GL}\left(\mathbb{R}^{[0,+\infty[}\right)$ maps elements of the Lie algebra on endomorphisms for the bundle $\mathcal{V}$. Given a local cash flow section $f_{t}=\int_{0}^{+\infty} d s f_{s} \delta_{s-t}$, in $\left.\mathcal{V}\right|_{U}$ and a local vector field $X$ in $\left.T M\right|_{U}$, the connection $\nabla^{\mathcal{V}}$ has the following local representation:

$$
\nabla_{X}^{\mathcal{V}} f_{t}=\int_{0}^{+\infty} d s\left(d f_{s}(X) \cdot v_{s}+f_{s} \omega(X) \cdot v_{s}\right),
$$

where $v_{s}:=\delta_{s-t}$ and $\omega$ is an element of $\left.T^{*} U\right|_{U} \otimes \mathcal{L}\left(\left.V\right|_{U}\right)$, i.e., an endomorphism valued 1 -form defined as follows:

$$
\omega(x)(X):=\left(T_{e} \rho \cdot \chi(x, e)\right) \cdot X=\left.\frac{d}{d \varepsilon}\right|_{\varepsilon=0} \rho(\exp (\varepsilon \chi(x, e) \cdot X) e) .
$$

Since the derivative of the exponential map is the identity and

$$
\rho(\pi)=\pi * \in \operatorname{GL}\left(\mathcal{V}_{x}\right) \Rightarrow T_{e} \rho . t=t * \cdot \in \mathcal{L}\left(\mathcal{V}_{x}\right),
$$

it follows that

$$
\omega(x)=\chi(x, e) * \cdot=K(x) \otimes \delta * \cdot,
$$

and therefore,

$$
\begin{aligned}
\nabla_{X}^{\mathcal{V}} f_{t} & =\int_{0}^{+\infty} d s\left[d f_{s}(X) v_{s}+f_{s} K \cdot X \delta * v_{s}\right] \\
& =\int_{0}^{+\infty} d s\left[d f_{s}(X)+f_{s} K \cdot X\right] v_{s} \\
& =d f_{t}(X)+f_{t} K \cdot X \\
& =\sum_{j=0}^{N}\left(\frac{\partial f_{t}}{\partial x_{j}}+K_{j} f_{t}\right) X_{j} .
\end{aligned}
$$


Proposition 5. The curvature of the connection $\nabla^{\mathcal{V}}$ is

$$
R^{\mathcal{V}}(X, Y):=\nabla_{X}^{\mathcal{V}} \nabla_{Y}^{\mathcal{V}}-\nabla_{Y}^{\mathcal{V}} \nabla_{X}^{\mathcal{V}}-\nabla_{[X, Y]}^{\mathcal{V}}=[p] \circ\left(R\left(X^{*}, Y^{*}, e\right) * \cdot\right) \circ[p]^{-1},
$$

where $R$ is the curvature on the principal fibre bundle $\mathcal{B}, X^{*}, Y^{*} \in T_{p} \mathcal{B}$ the horizontal lifts of $X, Y \in T_{(t, x)} M$, and

$$
\begin{aligned}
{[p]: V=\mathbb{R}^{[0,+\infty[} } & \longrightarrow \mathcal{V}_{(t, x)}:=\mathcal{B}_{(t, x)} \times{ }_{G} V \\
v & \longmapsto[p](v)=[p, v]
\end{aligned}
$$

is the fibre isomorphism between $\mathcal{B}$ and $\mathcal{V}$. In particular, the curvature on the principal fibre bundle vanishes if and only if the curvature on the associated vector bundle vanishes.

Proof. Equation (59) follows from the definition of the curvature on a vector bundle and utilizes Satz 3.21 in [26] with $\rho(\pi)=\pi * \cdot$.

We now continue by introducing the connection Laplacian on an appropriate Hilbert space.

Definition 16. The space of the sections of the cash flow bundle can be turned into a scalar product space by introducing, for stochastic sections $f=f(t, x, \omega)=\left(f_{s}(t, x, \omega)\right)_{s \in[0,+\infty[}$ and $g=g(t, x, \omega)=\left(g_{s}(t, x, \omega)\right)_{s \in[0,+\infty[}$, the following:

$$
\begin{aligned}
(f, g):=\int_{\Omega} d P \int_{X} d^{N} x \int_{0}^{+\infty} d t\langle f, g\rangle(t, x, \omega) & \left.=\mathbb{E}_{0}\left[(f, g)_{L^{2}(M, \mathbb{R}}[0,+\infty]\right)\right] \\
& =(f, g)_{L^{2}\left(\Omega, \mathcal{V}, \mathcal{A}_{0}, d P\right)}
\end{aligned}
$$

where

$$
\langle f, g\rangle(x, t, \omega):=\int_{0}^{+\infty} d s f_{s}(t, x, \omega) g_{s}(t, x, \omega) .
$$

The Hilbert space of integrable sections reads as follows:

$$
\begin{aligned}
\mathcal{H} & :=L^{2}\left(\Omega, \mathcal{V}, \mathcal{A}_{0}, d P\right) \\
& =\left\{f=f(t, x, \omega)=\left(f_{s}(t, x, \omega)\right)_{s \in[0,+\infty[} \mid(f, f)_{L^{2}\left(\Omega, \mathcal{V}, \mathcal{A}_{0}, d P\right)}<+\infty\right\} .
\end{aligned}
$$

When considering the connection Laplacian, there are two standard choices for a local elliptic boundary condition which guarantees self-adjointness:

- Dirichlet boundary condition:

$$
B_{D}(f):=\left.f\right|_{\partial M}
$$

- Neumann boundary condition:

$$
B_{N}(f):=\left.\left(\nabla_{v}^{\mathcal{V}} f\right)\right|_{\partial M},
$$

where $v$ denotes the normal unit vector field to $\partial M$.

By considering the $\omega$ a parameter dependence, we can apply a standard result functional analysis to obtain the following proposition.

Proposition 6. The connection Laplacian $\Delta^{\mathcal{V}}:=\nabla^{\mathcal{V}} \nabla^{\mathcal{V}}$ with a domain definition given by the Neumann boundary condition,

$$
\operatorname{dom}\left(\Delta_{B_{N}}^{\mathcal{V}}\right):=\left\{f \in \mathcal{H} \mid f(\omega, \cdot, \cdot) \in H^{2}\left(M, \mathbb{R}^{[0,+\infty[}\right), B_{N}(f(\omega, \cdot, \cdot))=0 \forall \omega \in \Omega\right\},
$$


is a self-adjoint operator on $\mathcal{H}$. Its spectrum consists in the disjoint union of the discrete spectrum (eigenvalues) and the continuous spectrum (approximate eigenvalues) lying in $[0,+\infty[$ :

$$
\operatorname{spec}\left(\Delta_{B_{N}}^{\mathcal{V}}\right)=\operatorname{spec}_{d}\left(\Delta_{B_{N}}^{\mathcal{V}}\right) \cup \operatorname{Uspec}_{c}\left(\Delta_{B_{N}}^{\mathcal{V}}\right)
$$

If $M$ is compact, for example, by setting $M:=[0, T] \times \mathfrak{X}, \mathfrak{X} \subset \mathbb{R}^{N}$ compact and $T<+\infty$, then the continuous spectrum is empty and the eigenvalues can be ordered in a monotone increasing sequence converging to $+\infty$.

Remark 8. When we choose $M=[0, T] \times \mathfrak{X}$ with $T<+\infty$, we have to adapt the construction of the principal fibre bundle and the associated vector bundle accordingly. Note that the structure group of $\mathcal{B}$ and its Lie Algebra remain $G$ and $\mathbb{R}^{[0,+\infty[}$, respectively, and the fibre of $\mathcal{V}$ is still $\mathbb{R}^{[0,+\infty[}$. Only the integration over the time dimension in the base space $M$ is performed until $T$.

Remark 9. For a fixed $\omega \in \Omega$, the definition domain of $\Delta_{B_{N}}^{\mathcal{V}}$ is a subset of the Sobolev space $H^{2}\left(M, \mathbb{R}^{[0,+\infty[}\right)$. If $M$ is compact, then the eigenvectors of $\Delta_{B_{N}}^{\mathcal{V}}$ lie in $C^{\infty}\left(M, \mathbb{R}^{[0,+\infty[}\right)$ and satisfy the Neumann boundary condition. Proposition 6 follows from standard elliptic spectral theory by means of an integration over $\Omega$.

The spectrum of the connection Laplacian under the Neumann boundary condition contains information about arbitrage possibilities in the market.

Theorem 11. The market model satisfies the NFLVR condition if and only if $0 \in \operatorname{spec}_{d}\left(\Delta_{B_{N}}^{\mathcal{V}}\right)$. The harmonic sections parameterize the Radon-Nikodym derivative for the change of measure from the statistical to the risk-neutral measures.

Proof. The spectrum of the Laplacian under Neumann boundary conditions contains 0 if and only if there exists a section $f$ such that

$$
\nabla^{\mathcal{V}} f=0
$$

According to Proposition 4, this is equivalent to

$$
\frac{\partial f_{t}}{\partial x_{j}}+K_{j} f_{t}=0,
$$

for all $j=0,1, \ldots, N$. This means that for $j=0$

$$
\mathcal{D} \log \left(f_{t}(x)\right)-r_{t}^{x}=0,
$$

and for $j=1, \ldots, N$,

$$
\frac{\partial \log \left(f_{t}(x)\right)}{\partial x_{j}}=-\frac{D_{t}^{j}}{D_{t}^{x}},
$$


for all $x \in \mathfrak{X}$. Equation (70) becomes

$$
\begin{aligned}
& \frac{\partial \log \left(f_{t}(x)\right)}{\partial x_{j}}=-\frac{\partial \log \left(D_{t}^{x}\right)}{\partial x_{j}} \\
& \frac{\partial \log \left(f_{t}(x)\right) D_{t}^{x}}{\partial x_{j}}=0 \\
& \left.\log \left(f_{t}(x)\right) D_{t}^{x}\right) \equiv C_{t} \\
& f_{t}(x) D_{t}^{x} \equiv \exp \left(C_{t}\right),
\end{aligned}
$$

for a process $\left(C_{t}\right)_{t \in[0,+\infty[}$. Therefore, the positive process $\beta=\left(\beta_{t}\right)_{t \in[0,+\infty[}:=\left(\exp \left(-C_{t}\right)\right)$ $t \in[0,+\infty[$ satisfies

$$
f_{t}(x)=\frac{1}{\beta_{t} D_{t}^{x}},
$$

which, when inserted into Equation (69), leads to

$$
\mathcal{D} \log \left(\beta_{t} D_{t}^{x}\right)+r_{t}^{x}=0,
$$

for all $t$ and $x$.

For fixed $\omega \in \Omega$, the Laplace operator has an elliptic symbol and by Weyl's theorem, any harmonic $f=f(\omega, t, x)$ is a smooth function of $(t, x)$. In particular, any path of $f$ is càdlàg with bounded variation, and hence $\left(f_{t}\right)_{t}$ is a semimartingale. According to Equation (72), $\left(D_{t}\right)_{t}$ being a semimartingale, it follows that $\left(\beta_{t}\right)_{t}$ is a semimartingale as well. Based on Theorem 7 this is equivalent to the NFLVR condition.

Remark 10. Note that if $f=f(\omega, t, x) \equiv f(\omega)$, and at least one of the components of $r$ or $D$ does not vanish, then $f=0,0 \notin \operatorname{spec}\left(\Delta_{B_{N}}^{\mathcal{V}}\right)$, confirming and extending Remark 5 .

Remark 11. Any harmonic $f=f_{t}(x)$ defines a risk-neutral measure by means of the RadonNikodym derivative:

$$
\frac{d P^{*}}{d P}=\frac{\beta_{t}}{\beta_{0}}=\frac{D_{0}^{x}}{D_{t}^{x}} \frac{f_{0}(x)}{f_{t}(x)}
$$

which does not depend on $x$.

From Formula (74), we derive the following corollary.

Corollary 2. The market model is complete if and only if $0 \in \operatorname{spec}\left(\Delta^{\mathcal{V}}\right)_{B_{N}}$ is an eigenvalue with simple multiplicity.

Remark 12. The situation for the Dirichlet boundary condition is similar. The proposition and remark analogous to Proposition 6 and Remark 9 hold true. But because of the unique continuation property for elliptic operators, 0 never lies in $\operatorname{spec}_{d}\left(\Delta_{B_{D}}^{\mathcal{V}}\right)$ whether the NFLVR property is satisfied or not. 


\subsection{Arbitrage Bubbles}

Definition 17 (Spectral Lower Bound). The highest spectral lower bound of the connection Laplacian on the cash flow bundle $\mathcal{V}$ is given by the following:

$$
\lambda_{0}:=\inf _{\substack{\varphi \in C^{\infty}(M, \mathcal{V}) \\ \varphi \neq 0 \\ B_{N}(\varphi)=0}} \frac{\left(\nabla^{\mathcal{V}} \varphi, \nabla^{\mathcal{V}} \varphi\right)_{\mathcal{H}}}{(\varphi, \varphi)_{\mathcal{H}}},
$$

and it is assumed on the subspace

$$
E_{\lambda_{0}}:=\left\{\varphi \mid \varphi \in C^{\infty}(M, \mathcal{V}) \cap \mathcal{H}, B_{N}(\varphi)=0,\left(\nabla^{\mathcal{V}} \varphi, \nabla^{\mathcal{V}} \varphi\right)_{\mathcal{H}} \geq \lambda_{0}(\varphi, \varphi)_{\mathcal{H}}\right\} .
$$

The space

$$
\mathcal{K}_{\lambda_{0}}:=\left\{\varphi \in E_{\lambda_{0}} \mid \varphi \geq 0, \mathbb{E}[\varphi]=1\right\}
$$

contains all candidates for the Radon-Nikodym derivative

$$
\frac{d P^{*}}{d P}=\varphi,
$$

for a probability measure $P^{*}$ absolutely continuous with respect to the statistical measure $P$.

By reformulating Theorem 11 and Corollary 2, we obtain following statement.

Proposition 7. The market model satisfies the NFLVR condition if and only if $\lambda_{0}=0$. Therefore, there exist risk-neutral probability measures defined by (78) with the corresponding $\varphi \in \mathcal{K}_{\lambda_{0}}$ such that $\left(D_{t}\right)_{t \in[0, T]}$ is a vector valued martingale with respect to $P^{*}$, i.e.,

$$
\mathbb{E}_{t}^{*}\left[D_{s}\right]=D_{t} \quad \text { for all } s \geq \operatorname{tin}[0, T] .
$$

The market is complete if and only if $\lambda_{0}=0$ and $\operatorname{dim} E_{0}=1$.

For arbitrage markets we have $\lambda_{0}>0$, and there exist no risk-neutral probability measures. Nevertheless, it is possible to define a fundamental value, although not in a unique way.

Definition 18 (Basic Assets' Arbitrage Fundamental Prices and Bubbles). Let $\left(C_{t}\right)_{t \in[0, T]}$ be the $\mathbb{R}^{N}$ cash flow stream stochastic process associated with the $N$ assets of the market model with a given spectral lower bound $\lambda_{0}$ and the Radon-Nikodym subspace $\mathcal{K}_{\lambda_{0}}$. For a given choice of $\varphi \in \mathcal{K}_{\lambda_{0}}$, the approximated fundamental value of the assets with a stochastic $\mathbf{R}^{N_{-} \text {-valued price }}$ process $\left(S_{t}\right)_{t \in[0, T]}$ is defined as follows:

$$
S_{t}^{*, \varphi}:=\mathbb{E}_{t}\left[\varphi\left(\int_{t}^{\tau} d C_{u} \exp \left(-\int_{t}^{u} r_{s}^{0} d s\right)+S_{\tau} \exp \left(-\int_{t}^{\tau} r_{s}^{0} d s\right) 1_{\{\tau<+\infty\}}\right)\right] 1_{\{t<\tau\}},
$$

where $\tau$ denotes the maturity time of all risky assets in the market model, and the approximated bubble is defined as follows:

$$
B_{t}^{\varphi}:=S_{t}-S_{t}^{*, \varphi}
$$


The fundamental price vector for the assets and their asset bubble prices are defined as:

$$
\begin{aligned}
& S_{t}^{*}:=\tilde{S}_{t}^{*, \varphi_{0}} \\
& B_{t}:=B_{t}^{\varphi_{0}} \\
& \varphi_{0}:=\arg \min _{\varphi \in \mathcal{K}_{\lambda_{0}}} \mathbb{E}_{0}\left[\int_{0}^{T} d s\left|B_{s}^{\varphi}\right|^{2}\right] .
\end{aligned}
$$

The probability measure $P^{*}$ with the Radon-Nikodym derivative

$$
\frac{d P^{*}}{d P}=\varphi_{0}
$$

is termed minimal arbitrage measure.

Proposition 8. The assets' fundamental values can be expressed as conditional expectation with respect to the minimal arbitrage measure using the following formula:

$$
S_{t}^{*}:=\mathbb{E}_{t}^{*}\left[\int_{t}^{\tau} d C_{u} \exp \left(-\int_{t}^{u} r_{s}^{0} d s\right)+S_{\tau} \exp \left(-\int_{t}^{\tau} r_{s}^{0} d s\right) 1_{\{\tau<+\infty\}}\right] 1_{\{t<\tau\}} .
$$

Definition 19 (Scalar curvature). The market integral scalar curvature at time $t$ for the portfolio $x$ is defined as follows:

$$
\mathcal{K}(t, x):=\mathcal{D} \log D_{t}^{x}+r_{t}^{x}
$$

A strategy $x=\left(x_{t}\right)_{t \in[0, T]}$ is a free lunch / no-arbitrage / rip-off strategy if and only if

$$
\mathcal{K}\left(t, x_{t}\right)\left\{\begin{array}{ll}
>0 & \text { (free lunch) } \\
=0 & \text { (no-arbitrage) } \\
<0 & \text { (rip off) }
\end{array} \quad \text { for all } \quad t \in[0, T] .\right.
$$

The vector valued integral curvature of the portfolio is defined as the vector of integral scalar curvatures for the portfolio single asset components:

$$
\overrightarrow{\mathcal{K}}(t, x):=\sum_{j=1}^{N}\left(\mathcal{D} \log D_{t}^{x^{j} e_{j}}+r_{t}^{x^{j} e_{j}}\right) e_{j}
$$

Remark 13. The curvature $R$ defined in (29) can be written as follows:

$$
R(t, x, g)=g d t \wedge d_{x} \mathcal{K}(t, x),
$$

therefore justifying the nomenclature of the integral scalar curvature $\mathcal{K}$.

We can now extend Jarrow-Protter-Shimbo's result in [27] to obtain the following bubble decomposition theorem.

Theorem 12 (Bubble decomposition). Let $\tau$ denote the maturity time of all risky assets in the market model. $S_{t}$ admits a unique (up to the P-evanescent set) decomposition into a sum of fundamental and bubble values:

$$
S_{t}=S_{t}^{*}+B_{t}
$$


where $\left(B_{t}\right)_{t \in[0, T]}$ is a càdlàg process satisfying

$$
\begin{aligned}
& B_{t}=S_{t}+ \\
& \quad-\mathbb{E}_{t}\left[\varphi _ { 0 } \left\{\int_{t}^{\tau} d C_{u} \exp \left(-\int_{t}^{u} d s r_{s}^{0}\right)+\right.\right. \\
& \left.\left.\quad+S_{t} \exp \left(\int_{t}^{\tau} d s\left(\overrightarrow{\mathcal{K}}(s, e)-r_{s}^{0}\right)\right) 1_{\{\tau<+\infty\}}\right\}\right] 1_{\{t<\tau\}},
\end{aligned}
$$

where $e=[1, \ldots, 1]^{\dagger}$ or, equivalently, for all $j=1, \ldots, N$

$$
B_{t}^{j}=S_{t}^{j}-\mathbb{E}_{t}^{*}\left[\int_{t}^{\tau} d C_{u}^{j} \exp \left(-\int_{t}^{u} d s r_{s}^{0}\right)+\exp \left(-\int_{t}^{\tau} d s r_{s}^{0}\right) S_{\tau}^{j} 1_{\{\tau<+\infty\}}\right] 1_{\{t<\tau\}} .
$$

If all asset maturities are finite, i.e., $\tau=T<+\infty$, then

$$
\begin{aligned}
& \overrightarrow{\mathcal{K}} .>r^{0} \text { and } C .>0 \Longrightarrow B_{t} \uparrow 0^{-}\left(t \rightarrow T^{-}\right) \\
& \overrightarrow{\mathcal{K}} .<r^{0} \text { and } C .<0 \Longrightarrow B_{t} \downarrow 0^{+}\left(t \rightarrow T^{-}\right) \\
& \overrightarrow{\mathcal{K}} .=r^{0} \text { and } C .=0 \Longrightarrow B_{t} \equiv 0
\end{aligned}
$$

where the inequalities and the limits are meant componentwise.

Proof. By developing the expression for the bubbles' values and utilizing the definition of deflators for all $t \geq 0$

$$
D_{t}=\exp \left(-\int_{0}^{t} d s r_{s}^{0}\right) S_{t}
$$

since the curvature is the instantaneous asset portfolio log return (see [11])

$$
D_{\tau}=D_{t} \exp \left(\int_{t}^{\tau} d s\left(\mathcal{D} \log D_{s}+r_{s}\right)\right)
$$

we obtain

$$
S_{\tau}=S_{t} \exp \left(\int_{t}^{\tau} d s\left(\mathcal{D} \log D_{s}+r_{s}-r_{s}^{0}\right)\right)
$$

and hence,

$$
\begin{aligned}
B_{t}=S_{t}-\mathbb{E}_{t}\left[\varphi _ { 0 } \left\{\int_{t}^{\tau} d C_{u} \exp \left(-\int_{t}^{u} d s r_{s}^{0}\right)+\right.\right. \\
\left.\left.+S_{\tau} \exp \left(-\int_{t}^{\tau} d s r_{s}^{0}\right) 1_{\{\tau<+\infty\}}\right\}\right] 1_{\{t<\tau\}}= \\
=S_{t}-\mathbb{E}_{t}\left[\varphi _ { 0 } \left\{\int_{t}^{\tau} d C_{u} \exp \left(-\int_{t}^{u} d s r_{s}^{0}\right)+\right.\right. \\
\left.\left.+S_{t} \exp \left(\int_{t}^{\tau} d s\left(\overrightarrow{\mathcal{K}}(s, e)-r_{s}^{0}\right)\right) 1_{\{\tau<+\infty\}}\right\}\right] 1_{\{t<\tau\}},
\end{aligned}
$$

which is (90), and for finite $T$, it becomes 


$$
\begin{aligned}
& B_{t}=S_{t}-\mathbb{E}_{t} {\left[\varphi _ { 0 } \left(\int_{t}^{T} d C_{u} \exp \left(-\int_{t}^{u} d s r_{s}^{0}\right)+\right.\right.} \\
&\left.+S_{t} \exp \left(\int_{t}^{T} d s\left(\overrightarrow{\mathcal{K}}(s, e)-r_{s}^{0}\right)\right)\right]= \\
&=S_{t}-S_{t} \underbrace{\mathbb{E}_{t}\left[\varphi_{0} \exp \left(\int_{t}^{T} d s\left(\overrightarrow{\mathcal{K}}(s, e)-r_{s}^{0}\right)\right)\right]}_{=: A_{1}(t, T)}+ \\
&-\underbrace{\underbrace{}_{\mathbb{E}_{t}\left[\varphi_{0}\left(\int_{t}^{T} d C_{u} \exp \left(-\int_{t}^{u} d s r_{s}^{0}\right)\right)\right]}}_{=: A_{2}(t, T)} .
\end{aligned}
$$

Now, since $\mathbb{E}_{0}\left[\varphi_{0}\right]=1$, we observe that

$$
\begin{aligned}
\overrightarrow{\mathcal{K}} .-r^{0}>0, C .>0 \Longrightarrow & A_{1}(t, T)>0, A_{2}(t, T)>0, \\
& A_{1}(t, T) \downarrow 1^{+}, A_{2}(t, T) \downarrow 0^{+} \quad\left(t \rightarrow T^{-}\right) \\
\overrightarrow{\mathcal{K}} .-r^{0}<0, C .<0 \Longrightarrow & A_{1}(t, T)>0, A_{2}(t, T)<0, \\
& A_{1}(t, T) \uparrow 1^{+}, A_{2}(t, T) \uparrow 0^{+} \quad\left(t \rightarrow T^{-}\right),
\end{aligned}
$$

from which we conclude according to (97) that

$$
\begin{aligned}
& \overrightarrow{\mathcal{K}}_{t}-r_{t}^{0}>0 \text { for } t \in[0, T], C .>0 \Longrightarrow B_{t} \uparrow 0^{-}\left(t \rightarrow T^{-}\right) \\
& \overrightarrow{\mathcal{K}}_{t}-r_{t}^{0}<0 \text { for } t \in[0, T], C .<0 \Longrightarrow B_{t} \downarrow 0^{+}\left(t \rightarrow T^{-}\right),
\end{aligned}
$$

and hence,

$$
\overrightarrow{\mathcal{K}}_{t}-r_{t} \equiv 0 \text { and } C=0 \Longrightarrow B_{t} \equiv 0 \text {. }
$$

Inserting the definition (87) of $\overrightarrow{\mathcal{K}}$ into (90) leads to (91). The proof is now completed.

We can now extend Jarrow-Protter-Shimbo's result in [27] to obtain the following bubble classification theorem.

Theorem 13 (Bubble types). Let $T=+\infty$, and denote as $\tau$ the maturity time of all risky assets in the market model. If there exists a non-trivial bubble $B_{t}^{j}$ in an asset's price for $j=1, \ldots, N$, then there exists at least one probability measure $P^{*}$ equivalent to $P$, for which we have three and only three possibilities:

- $\quad$ Type1: $B_{t}^{j}$ is local super-or submartingale with respect to both $P$ and $P^{*}$, if $P[\tau=+\infty]>0$;

- Type2: $B_{t}^{j}$ is local super-or submartingale with respect to both $P$ and $P^{*}$, but not uniformly integrable super-or submartingale, if $B_{t}^{j}$ is unbounded but with $P[\tau<+\infty]=1$;

- Type3: $B_{t}^{j}$ is a strict local super-or sub-P-and $P^{*}$-martingale, if $\tau$ is a bounded stopping time.

Proof. This theorem is a direct consequence of a local application of Equation (97), applied on time subintervals of $[0, T]$ on which the signs of $\mathcal{K}\left(t, e_{j}\right)+r_{t}^{0}$ and of $C_{t}$ remain constant. On the subintervals with non-negative $\mathcal{K}\left(t, e_{j}\right)$ and $C_{t}$, the bubble price $B_{t}^{j}$ is a sub-martingale for both $P$ and $P^{*}$, because for $t \leq s$ that is near enough, $B_{t} \leq B_{s}$ holds true; thus,

$$
B_{t} \leq \mathbb{E}_{t}\left[B_{s}\right] \text { and } \quad B_{t} \leq \mathbb{E}_{t}^{*}\left[B_{s}\right]
$$

On the subintervals with non-positive $\mathcal{K}\left(t, e_{j}\right)$ and $C_{t}$, the bubble price $B_{t}^{j}$ is a supermartingale for both $P$ and $P^{*}$, because for $t \leq s$ that is near enough, $B_{t} \geq B_{s}$ holds true; thus,

$$
B_{t} \geq \mathbb{E}_{t}\left[B_{s}\right] \quad \text { and } \quad B_{t} \geq \mathbb{E}_{t}^{*}\left[B_{s}\right]
$$


In the case of type 1 , there is a non-evanescent set of elementary events for which the maturity time of the assets is not finite, and without further information, we do not know whether the stochastic integral until infinite converges. In the case of type 2, the set of elementary events for which the maturity time of the assets $\tau$ is not finite vanishes a.s., but if $B_{t}^{j}$ is unbounded, we do not know if the stochastic integral over $[t, \tau]$ converges uniformly in $\tau$. In the case of type 3 , the stochastic integral converges. The proof is completed.

Remark 14. Note that if the NFLVR is satisfied, then the curvature vanishes, and so do the bubbles of type 3, these being trivial martingales for both the statistical and the risk-neutral probability measures.

Definition 20 (Contingent Claim's Arbitrage Fundamental Price and Bubble). Let us consider in the context of Definition (18) a European option given by the contingent claim with a unique payoff $H\left(S_{T}\right)$ at time $T$ for an appropriate real-valued function $H$ of $N$ real variables. The fundamental price of the contingent claim and its corresponding arbitrage bubble is defined in the case of base assets paying no dividends:

$$
\begin{aligned}
V_{t}^{*}(H) & :=\mathbb{E}_{t}\left[\varphi_{0} \exp \left(-\int_{t}^{T} r_{s}^{0} d s\right) H\left(S_{T}\right) 1_{\{T<+\infty\}}\right] 1_{\{t<T\}} \\
& =\mathbb{E}^{*}\left[\exp \left(-\int_{t}^{T} r_{s}^{0} d s\right) H\left(S_{T}\right) 1_{\{T<+\infty\}}\right] 1_{\{t<T\}} \\
B_{t}(H) & :=V_{t}(H)-V_{t}^{*}(H)
\end{aligned}
$$

where $\varphi_{0}$ is the minimizer for the basic assets bubbled defined in (82), $P^{*}$ the minimal arbitrage measure, and $\left(V_{t}(H)\right)_{t \in[0, T]}$ is the price process of the European option.

In the case of base assets paying dividends, the definition becomes the following:

$$
\begin{aligned}
V_{t}^{*}(H) & :=\mathbb{E}_{t}\left[\varphi_{0} \exp \left(-\int_{t}^{T} r_{s}^{0} d s\right) H\left(S_{T} \exp \left(\frac{C_{T}}{S_{T}}(T-t)\right)\right) 1_{\{T<+\infty\}}\right] 1_{\{t<T\}} \\
& =\mathbb{E}^{*}\left[\exp \left(-\int_{t}^{T} r_{s}^{0} d s\right) H\left(S_{T} \exp \left(\frac{C_{T}}{S_{T}}(T-t)\right)\right) 1_{\{T<+\infty\}}\right] 1_{\{t<T\}} \\
B_{t}(H) & :=V_{t}(H)-V_{t}^{*}(H),
\end{aligned}
$$

where $\frac{C_{t}^{j}}{S_{t}^{j}}$ is the instantaneous dividend rate for the $j$-th asset.

Remark 15. If the market is complete, then $\lambda_{0}=0$ and $\mathcal{K}_{\lambda_{0}}=\left\{\varphi_{0}\right\}$, where $\varphi_{0}$ is the RadonNikodym derivative of the unique risk-neutral probability measure with respect to the statistical probability measure. The definitions in (18) and in (12) for the complete market coincide with the definitions of fundamental value and asset bubble price for both base asset and contingent claim as introduced by Jarrow, Protter, and Shimbo in [27], proving that they are a natural extension to markets allowing for arbitrage opportunities.

Remark 16. We see that the fundamental price of an asset defined via minimal arbitrage measure does share common characteristics with the real world pricing in the benchmark approach by Platen and Heath (see chapters 9 and 10 of [28]).

We now prove the put-call parity of fundamental prices.

Proposition 9 (Put-Call Parity for Fundamental Prices). Let us consider the market model with $N=1$ for the base assets (i.e., cash and one risky asset). Then, the fundamental price processes:

- $\quad\left(C_{t}^{*}\right)_{t \in[0, T]}$ of a call option on $\left(S_{t}\right)_{t \in[0, T]}$ with strike price $K>0$ at time $T$;

- $\quad\left(P_{t}^{*}\right)_{t \in[0, T]}$ of a put option on $\left(S_{t}\right)_{t \in[0, T]}$ with strike price $K>0$ at time $T$; 
- $\quad\left(F_{t}^{*}\right)_{t \in[0, T]}$ of a forward on $\left(S_{t}\right)_{t \in[0, T]}$ with forward price $K>0$ at time $T$;

satisfy the put-call-parity relation if the base asset pays no dividends.

$$
C_{t}^{*}-P_{t}^{*}=F_{t}^{*}
$$

Proof. For a positive strike price $K>0$, we can decompose the forward payoff as:

$$
S_{T}-K=\left(S_{T}-K\right)^{+}-\left(K-S_{T}\right)^{+} \text {. }
$$

Formula (103), in the case of no dividends $(C \equiv 0)$, reads as:

$$
V_{t}^{*}(H)=\mathbb{E}_{t}\left[\varphi_{0} \exp \left(-\int_{t}^{T} r_{s}^{0} d s\right) H\left(S_{T}\right) 1_{\{T<+\infty\}}\right] 1_{\{t<T\}},
$$

which, applied to (106), leads to

$$
V_{t}^{*}\left(\left(S_{T}-K\right)^{+}\right)-V_{t}^{*}\left(\left(K-S_{T}\right)^{+}\right)=V_{t}^{*}\left(\left(S_{T}-K\right)\right),
$$

which is Equation (105). The proof is completed.

Remark 17. The put-call parity for market prices may be violated even under the NFLVR assumption (see [29]).

Finally, a short direct computation shows the corollary below.

Corollary 3. The bubble discounted values for the base assets in Definition 18 and for the contingent claim on the base assets paying dividends in Definition 12

$$
\widehat{B}_{t}:=\exp \left(-\int_{0}^{t} r_{s}^{0} d s\right) B_{t}, \quad \widehat{B}(H)_{t}:=\exp \left(-\int_{0}^{t} r_{s}^{0} d s\right) B(H)_{t}
$$

satisfy the equalities

$$
\begin{aligned}
& \widehat{B}_{t}^{j}=D_{t}^{j}-\left(\mathbb{E}_{t}^{*}\left[D_{\tau}^{j} 1_{\{\tau<+\infty\}}\right]+\mathbb{E}_{t}^{*}\left[\widehat{C}_{\tau}^{j} 1_{\{\tau<+\infty\}}\right]-\widehat{C}_{t}^{j}\right) 1_{\{t<\tau\}} \\
& \widehat{B}_{t}(H)=\widehat{V}_{t}(H)-\mathbb{E}_{t}^{*}\left[\widehat{H}\left(S_{T} \exp \left(\frac{C_{T}}{S_{T}}(T-t)\right)\right) 1_{\{T<+\infty\}}\right] 1_{\{t<T\}} .
\end{aligned}
$$

where

$$
\begin{aligned}
\widehat{C}_{t}^{j} & :=\exp \left(-\int_{0}^{t} r_{s}^{0} d s\right) C_{t}^{j} \\
\widehat{H} & :=\exp \left(-\int_{0}^{T} r_{s}^{0} d s\right) H \\
\widehat{V}_{t}(H) & :=\exp \left(-\int_{0}^{t} r_{s}^{0} d s\right) V_{t}(H)
\end{aligned}
$$

are the discounted cash flow for the $j$-th asset, the discounted contingent claim payoff, and the discounted value of the derivative.

\section{Topological Obstructions to Arbitrage}

\subsection{Topological Obstruction to Arbitrage Induced by the Gauss-Bonnet-Chern Theorem}

We want to show that the exterior algebra bundle twisted with the cash flow bundle defined in Section 3.1 can be given the structure of a Dirac bundle. Then, we apply the version of the Atiyah-Singer index theorem for this bundle, called the Gauss-Bonnet-Chern theorem, which relates the integral of the Euler form (i.e., the Pfaffian of the curvature of 
the connection) with a topological invariant of the bundle, the Euler characteristic. First, we recall some basic definitions and examples about Dirac operators.

Definition 21. The quadruple $(W,\langle\cdot, \cdot\rangle, \nabla, \gamma)$, where

(i) W is a complex (real) vector bundle over the oriented Riemannian manifold $(M, g)$ with a Hermitian (Riemannian) structure $\langle\cdot, \cdot\rangle$;

(ii) $\nabla: C^{\infty}(M, W) \rightarrow C^{\infty}\left(M, T^{*} M \otimes W\right)$ is a connection on $M$;

(iii) $\gamma: \mathrm{Cl}(M, g) \rightarrow \operatorname{Hom}(W)$ is a real algebra bundle homomorphism from the Clifford bundle over $M$ to the real bundle of complex (real) endomorphisms of $W$, i.e., $W$ is a bundle of Clifford modules;

is said to be a Dirac bundle if the following conditions are satisfied:

(iv) $\gamma(v)^{*}=-\gamma(v), \forall v \in T M$, i.e., the Clifford multiplication by tangent vectors is fiberwise skew-adjoint with respect to the Hermitian (Riemannian) structure $\langle\cdot, \cdot\rangle$;

(v) $\nabla\langle\cdot, \cdot\rangle=0$, i.e., the connection is Leibnizian (Riemannian). In other words, it satisfies the product rule:

$$
d\langle\varphi, \psi\rangle=\langle\nabla \varphi, \psi\rangle+\langle\varphi, \nabla \psi\rangle, \quad \forall \varphi, \psi \in C^{\infty}(M, W)
$$

(vi) $\nabla \gamma=0$, i.e., the connection is a module derivation. In other words, it satisfies the product rule:

$$
\begin{aligned}
\nabla(\gamma(w) \varphi)=\gamma\left(\nabla^{g} w\right) \varphi+\gamma(w) \nabla \varphi, & \forall \varphi, \psi \in C^{\infty}(M, W), \\
& \forall w \in C^{\infty}(M, C l(M, g)) .
\end{aligned}
$$

Example 1. (Exterior algebra bundle as a Dirac Bundle). Let $(M, g)$ be a $C^{\infty}$ Riemannian manifold of dimension $m$. The tangent and the cotangent bundles are identified by the b-map defined by $v^{b}(w):=g(v, w)$. Its inverse is denoted as $\sharp$. The exterior algebra can be seen as a Dirac bundle after the following choices:

- $W:=\Lambda\left(T^{*} M\right)=\bigoplus_{j=0}^{m} \Lambda^{j}\left(T^{*} M\right)$ : exterior algebra over $M$;

- $\langle\cdot, \cdot\rangle:$ Riemannian structure induced by $g$;

- $\quad \nabla$ : lift of the Levi Civita connection;

- By means of interior and exterior multiplication, $\operatorname{int}(v) \varphi:=\varphi(v, \cdot)$ and $\operatorname{ext}(v) \varphi:=v^{b} \wedge \varphi$, we can define the following:

$$
\begin{aligned}
T M & \longrightarrow \operatorname{Hom}(W) \\
v & \longmapsto \gamma(v):=\operatorname{ext}(v)-\operatorname{int}(v) .
\end{aligned}
$$

Recall that, since $\gamma^{2}(v)=-g(v, v) \mathbf{1}$, based on the universal property, the map $\gamma$ extends uniquely to a real algebra bundle endomorphism $\gamma: \mathrm{Cl}(M, g) \longrightarrow \operatorname{Hom}(W)$.

Definition 22. Let $(W,\langle\cdot, \cdot\rangle, \nabla, \gamma)$ be a Dirac bundle over the Riemannian manifold $(M, g)$. The Dirac operator $Q: C^{\infty}(M, W) \rightarrow C^{\infty}(M, W)$ is defined by $Q:=\gamma \circ(\sharp \otimes \nVdash) \circ \nabla$

$$
\begin{array}{cc}
C^{\infty}(M, W) \stackrel{\nabla}{\longrightarrow} C^{\infty}\left(M, T^{*} M \otimes W\right) \\
Q:=\gamma \circ(\sharp \otimes \nVdash) \circ \nabla \downarrow & \downarrow \sharp \otimes \nVdash \\
C^{\infty}(M, W) \stackrel{\gamma}{\longleftarrow} C^{\infty}(M, T M \otimes W)
\end{array}
$$

The square of the Dirac operator $P:=Q^{2}: C^{\infty}(M, W) \rightarrow C^{\infty}(M, W)$ is called the Dirac Laplacian. 
Definition 23 (Dirac Complex). Let $Q$ be the Dirac operator for the Dirac bundle $W$ over the Riemannian manifold $(M, g)$ and $T \in \operatorname{Hom}(W) .(Q, T)$ is called a Dirac complex if and only if $T^{2}=\mathbf{1}$ and $Q T=-T Q$. We introduce the following notation:

$$
\Pi_{ \pm}:=\frac{\mathbf{1} \mp T}{2} \quad W_{ \pm}:=\Pi_{ \pm}(W) \quad Q_{ \pm}:=\left.Q\right|_{C^{\infty}\left(M, W_{ \pm}\right)} .
$$

Remark 18. The terminology introduced in Definition 23 is justified by the following properties:

- $Q_{ \pm}: C^{\infty}\left(M, W_{ \pm}\right) \longrightarrow C^{\infty}\left(M, W_{\mp}\right)$;

- $Q=\left[\begin{array}{cc}0 & Q_{-} \\ Q_{+} & 0\end{array}\right]: C^{\infty}(M, \underbrace{W_{+} \oplus W_{-}}_{W}) \longrightarrow C^{\infty}(M, \underbrace{W_{+} \oplus W_{-}}_{W}) ;$

- $\quad$ the sequence

$$
0 \longrightarrow C^{\infty}\left(M, W_{+}\right) \stackrel{Q_{+}}{\longrightarrow} C^{\infty}\left(M, W_{-}\right) \stackrel{Q_{-}}{\longrightarrow} C^{\infty}\left(M, W_{+}\right) \longrightarrow 0
$$

is a complex, i.e., $Q_{-} Q_{+}=0$.

Example 2. (Exterior algebra bundle as a Dirac Bundle-Continuation). The Dirac operator $Q=d+\delta$ is termed a Euler operator. We define the vector bundle isomorphism on the exterior algebra bundle $T$ as $T \eta:=(-1)^{j} \eta$ for $\eta \in \Lambda^{j}\left(T^{*} M\right)$ and extend it by linearity to $\Lambda\left(T^{*} M\right)$ in order to obtain the Dirac complex $(Q, T)$, termed the rolled-up De Rham complex.

The Dirac operator $Q$ is elliptic and symmetric as an operator on the Hilbert space $L^{2}(M, W)$, with the appropriate choice of domain of definition.

Definition 24 (Analytical Index). Let $(Q, T)$ be a Dirac complex over a compact manifold. If $\partial M=\varnothing$, then the analytical index of the complex is defined as follows:

$$
\operatorname{Index}_{a}(Q, T):=\operatorname{dim} \operatorname{ker}\left(Q_{+}\right)-\operatorname{dim} \operatorname{ker}\left(Q_{-}\right) .
$$

If $\partial M \neq \varnothing$, and there exists an elliptic boundary condition $B$ for which $Q_{B}$ is symmetric, then the analytical index of the complex with respect to this boundary condition is defined as follows:

$$
\operatorname{Index}_{a}(Q, T, B):=\operatorname{dim} \operatorname{ker}\left(\left(Q_{+}\right)_{B}\right)-\operatorname{dim} \operatorname{ker}\left(\left(Q_{-}\right)_{B}\right) .
$$

Theorem 14 (Atiyah-Patodi-Singer). Let $(Q, T)$ be a Dirac complex over a compact manifold. If $\partial M=\varnothing$, then

$$
\operatorname{Index}_{a}(Q, T)=\operatorname{Index}(Q, T),
$$

where Index $x_{t}(Q, T)$ is a topological index, i.e., depending only on the topology of $M$ and $W$. If $\partial M \neq \varnothing$ and $B$ is an elliptic boundary condition, then

$$
\operatorname{Index}_{a}(Q, T, B)=\operatorname{Index}(Q, T)+\text { Boundary } \operatorname{Term}(B) .
$$

For generic Dirac bundles, the existence of local elliptic boundary conditions is not guaranteed. But for the exterior algebra bundle, the absolute and relative boundary conditions are always local elliptic boundary conditions for the Euler operator. In this case, the Atiyah-Singer index theorem takes the form of the Gauss-Bonnet-Chern theorem:

Example 3. (Exterior algebra bundle as a Dirac Bundle-Continuation). In the boundaryless case we have (see [30] page 179 and [31] page 59)

$$
(2 \pi)^{\frac{m}{2}} \int_{M} P f\left(-R^{\Lambda\left(T^{*} M\right)}\right)=\chi(M),
$$


where $\operatorname{Pf}\left(-R^{\Lambda\left(T^{*} M\right)}\right)$, termed a Euler form, is the Pfaffian of the exterior algebra curvature and $\chi(M)$ is the Euler characteristic, which is a topological invariant of the manifold defined as:

$$
\chi(M):=\sum_{j=0}^{m} b_{j}(M)
$$

where $b_{j}(M):=\operatorname{dim} H^{j}(M)$ is the $j$-th Betti number of $M$, the dimension of the $j$-the De Rham absolute cohomology group, which is isomorphic to the $j$-th homology group. In the boundaryless case, absolute and relative cohomology coincide, the Hodge star operator defines an isomorphism between the $j$-th and the $m-j$-th De Rham cohomology, and hence, the Euler characteristic always vanishes if $m$ is odd.

If the manifold $M$ has a boundary on which we impose the absolute (or relative) boundary condition, the equality (122) becomes

$$
\left.(2 \pi)^{\frac{m}{2}} \int_{M} P f\left(-R^{\Lambda\left(T^{*} M\right)}\right)=\chi(M)+\int_{\partial M} \Phi\left(R^{\Lambda\left(T^{*} M\right)}, L(\partial M, M)\right)\right) d v o l_{\partial M},
$$

where $\Phi$ is a function of the curvature $R^{\Lambda\left(T^{*} M\right)}$ and of the second fundamental form $L$ of the embedding $\partial M \rightarrow M$ (see [30] page 201). Note that in the case of a manifold with a boundary case, the Euler characteristic does not have to vanish if $m$ is odd.

Next, we can apply the definitions above to recognize a Dirac bundle containing all the information required by the market model. In the space of all possible strategies $M:=[0, T] \times \mathfrak{X} \subset \mathbb{R}^{N+1}$, now we introduce the Riemannian structure induced by the Euclidean metric in $\mathbb{R}^{N+1}$. The cash flow bundle $\mathcal{V}$ has infinite rank and is therefore an unfavourable candidate for the Atiyah-Singer/Gauss-Bonnet-Chern theorem. Therefore, we choose a partition $t_{0}:=0<t_{1}<\cdots<t_{n-1}<t_{n}:=T$ of the interval $[0, t]$ such that its mesh $\max _{1<i<n}\left(t_{i}-t_{i-1}\right) \rightarrow 0(n \rightarrow+\infty)$ holds true. We repeat the construction of the market fibre bundle $\mathcal{B}_{n}$ in Definition 11, choosing as structure group $G_{n}:=\{\pi \in$ $\left.G \mid \operatorname{supp}(\pi) \subset\left\{t_{0}, \ldots, t_{n}\right\}\right\}$ with Lie algebra $\mathfrak{g}_{n}=\mathbb{R}^{n+1}$, and the construction of the cash flow bundle $\mathcal{V}_{n}$ in Section 3.1, choosing as fibre $V_{n}:=\mathbb{R}^{n+1}$. Note that in this case, by standard elliptic theory, the eigenspaces of the connection Laplacian under the Neumann boundary condition are all finite dimensional. In particular, there are finitely many linear, independent Radon-Nikodym derivatives that can perform a change of measure from the statistical to a risk-neutral one.

The exterior algebra bundle over $M$ twisted with the cash flow bundle $\mathcal{V}_{n}$ is given the structure of a Dirac bundle with the following choices:

- $g$ : restriction of the Euclidean metric;

- $W_{n}:=\Lambda\left(T^{*} M\right) \otimes \mathcal{V}_{n}$ : twisted bundle of finite rank $(n+1) 2^{N+1}$;

- $\left\langle\eta_{1} \otimes v_{1}, \eta_{2} \otimes v_{2}\right\rangle^{W_{n}}:=\left\langle\eta_{1}, \eta_{2}\right\rangle^{\Lambda\left(T^{*} M\right)}\left\langle v_{1}, v_{2}\right\rangle^{\mathcal{V}_{n}}$ : Riemannian structure;

- $\nabla^{W_{n}}:=\nabla^{\Lambda\left(T^{*} M\right)} \otimes \mathbf{1}_{\mathcal{V}_{n}}+\mathbf{1}_{\Lambda\left(T^{*} M\right)} \otimes \nabla^{\mathcal{V}_{n}}$ : connection;

- $\quad \gamma^{W_{n}}:=\gamma^{\Lambda\left(T^{*} M\right)} \otimes \mathbf{1}_{\mathcal{V}_{n}}:$ real algebra bundle endomorphism $\gamma: \mathrm{Cl}(M, g) \longrightarrow \operatorname{Hom}\left(W_{n}\right)$;

- $\quad T^{W_{n}}:=T^{\Lambda\left(T^{*} M\right)} \otimes \mathbf{1}_{\mathcal{V}_{n}} \in \operatorname{Hom}\left(W_{n}\right)$ : a symmetry anticommuting with the Dirac operator.

Properties (iv)-(vi) of Definition 21 are satisfied, as highlighted on page 226 of [30], and $T^{W_{n}}$ defines via Definition 23 a Dirac complex. Since for the curvature we have the following:

$$
R^{W_{n}}=R^{\Lambda\left(T^{*} M\right)} \otimes \mathbf{1}_{\mathcal{V}_{n}}+\mathbf{1}_{\Lambda\left(T^{*} M\right)} \otimes R^{\mathcal{V}_{n}}=\mathbf{1}_{\Lambda\left(T^{*} M\right)} \otimes R^{\mathcal{V}_{n}},
$$


because $M$ carries the flat Euclidean metric $\left(R^{\Lambda\left(T^{*} M\right)}=0\right)$, the Gauss-Bonnet-Chern theorem reads as follows:

$$
\begin{aligned}
(2 \pi)^{\frac{N+1}{2}} & \int_{M} \operatorname{Pf}\left(-\mathbf{1}_{\Lambda\left(T^{*} M\right)} \otimes R^{\mathcal{V}_{n}}\right) \\
& \left.=\operatorname{Rank}\left(\mathcal{V}_{n}\right)\left[\chi(M)+\int_{\partial M} \Phi\left(R^{\Lambda\left(T^{*} M\right)}, L(\partial M, M)\right)\right) d \operatorname{vol}_{\partial M}\right] .
\end{aligned}
$$

The integrand of the boundary term vanishes because the Riemannian curvature is zero, as one can see in [30] page 199. Therefore,

$$
(2 \pi)^{\frac{N+1}{2}} \int_{M} \operatorname{Pf}\left(-\mathbf{1}_{\Lambda\left(T^{*} M\right)} \otimes R^{\mathcal{V}_{n}}\right)=\operatorname{Rank}\left(\mathcal{V}_{n}\right) \chi(M) .
$$

Utilizing the fact that

$$
\chi(M)=\chi([0, T] \times \mathfrak{X})=\chi(\mathfrak{X}),
$$

and inserting the value for the cash flow bundle rank, we obtain for all $n \in \mathbb{N}_{1}$

$$
\frac{1}{n+1}\left(\frac{\pi}{2}\right)^{\frac{N+1}{2}} \int_{M} \operatorname{Pf}\left(-\mathbf{1}_{\Lambda\left(T^{*} M\right)} \otimes R^{\mathcal{V}_{n}}\right)=\chi(\mathfrak{X}),
$$

from which we see that a non-vanishing Euler characteristic of the space of all possible nominals is a topological obstruction for the market model to be arbitrage-free. To summarize:

Theorem 15. If a market model has a bounded space of asset nominals $\mathfrak{X}$, whose Euler characteristic $\chi(\mathfrak{X})$ does not vanish, then the zero curvature condition (ZC), or equivalently the (NUPBR) condition, and a fortiori the NFLVR condition, cannot be satisfied:

$$
(N F L V R) \Rightarrow(N U P B R) \Rightarrow \chi(\mathfrak{X})=0 .
$$

Remark 19. Formula (130) says that the total quantity of arbitrage allowed by a market over all asset strategies is a topological invariant of the asset nominal space.

Remark 20. The Euler characteristic of the nominal space $\chi(\mathfrak{X})$ as topological obstruction to NFLVR is consistent with the results in [10], where the first homotopy group $\Pi_{1}(\mathfrak{X}, D)$ must be trivial if NFLVR holds true.

Remark 21. There is a more general version of the index theorem which holds true for any elliptic operator on a vector bundle over a manifold with boundary that has an elliptic boundary condition, which is a generalization of the Atiyah-Singer-Patodi index theorem. For the connection Laplacian $P:=\nabla^{\mathcal{V}} \nabla^{\mathcal{V}}$ over the cash flow bundle $\mathcal{V}$ under the Neumann boundary condition $B_{N}$, it has the following form:

$$
\operatorname{Index}\left(P, B_{N}\right)=\operatorname{Index}(P)+\text { Boundary } \operatorname{Term}\left(P, B_{N}\right) .
$$

Do we expect from (132) another topological obstruction to arbitrage than the one induced by the Gauss-Bonnet-Chern theorem? We want to answer this question by means of qualitative reasoning. We observe that:

- $\quad \operatorname{Index}_{a}\left(P, B_{N}\right)$ is an integral over $M$ of an expression depending on the derivatives of Christoffel's symbols for the connection $\nabla^{\mathcal{V}}$;

- $\operatorname{Index}_{t}\left(P, B_{N}\right)$ is a topological invariant of the manifold $M$;

- $\quad \operatorname{Boundary} \operatorname{Term}\left(P, B_{N}\right)=0$, because of the choice of the boundary condition and Green's formula.

Therefore, the information contained in (132) is essentially the same as that in (125), and thus, we expect no additional insights. 


\subsection{Topological Obstruction to Arbitrage Induced by the Bochner-Weitzenböck Theorem}

Let us now introduce two local elliptic boundary condition which guarantee selfadjointness to the Dirac operator and the Dirac Laplacian on $\Lambda\left(T^{*} M\right)$ :

- Absolute boundary condition:

$$
\begin{aligned}
& B_{\mathrm{abs}}^{0}(f):=\left.(\operatorname{int}(v)(f))\right|_{\partial M} \\
& B_{\mathrm{abs}}^{1}(f):=B_{\mathrm{abs}}^{0}(f) \oplus B_{\mathrm{abs}}^{0}\left(Q^{\Lambda\left(T^{*} M\right)} f\right),
\end{aligned}
$$

where the operation int is the interior multiplication in $\Lambda\left(T^{*} M\right)$, defined as

$$
\operatorname{int}(v)(f):=f(v, \cdot) .
$$

\section{- Relative boundary condition:}

$$
\begin{aligned}
& B_{\text {rel }}^{0}(f):=\left.(\operatorname{ext}(v)(f))\right|_{\partial M} \\
& B_{\text {rel }}^{1}(f):=B_{\text {rel }}^{0}(f) \oplus B_{\text {rel }}^{0}\left(Q^{\Lambda\left(T^{*} M\right)} f\right),
\end{aligned}
$$

where the operation ext is the exterior multiplication in $\Lambda\left(T^{*} M\right)$, defined as

$$
\operatorname{ext}(v)(f):=v^{\#} \wedge f
$$

and \# is the isomorphism between $T M$ and $T^{*} M$.

Theorem 16. The following equations hold:

$$
\left(Q_{B_{a b s}^{0} \otimes B_{N}}^{W}\right)^{2}=\left(\nabla^{W^{*}} \nabla^{W}\right)_{B_{a b s}^{1} \otimes B_{N}}
$$

Moreover, the no-free-lunch-with-vanishing-risk condition is satisfied if and only if the Dirac Laplacian admits harmonic sections of the cash flow bundle:

$$
(N F L V R) \Longleftrightarrow 0 \in \operatorname{spec}_{d}\left(\left(Q^{W}\right)_{B_{a b s}^{1} \otimes B_{N}}^{2}\right)
$$

Proof. The first equation in (137) follows from the Bochner-Weitzenböck formula, which holds true for any Dirac bundle (cf. [32] page 155):

$$
\left(Q_{B_{\mathrm{abs}}^{0} \otimes B_{N}}^{W}\right)^{2}=\left(\nabla^{W^{*}} \nabla^{W}\right)_{B_{\mathrm{abs}}^{1} \otimes B_{N}}+\mathcal{R}^{W},
$$

where

$$
\mathcal{R}^{W}:=\sum_{\mu, v=0}^{N} \gamma^{W}\left(e_{\mu}\right) \gamma^{W}\left(e_{\nu}\right) R^{W}\left(e_{\mu}, e_{\nu}\right)
$$

is the curvature homomorphism on the vector bundle $W$ and is independent of the choice of an o.n. frame of TM. Inserting

$$
R^{W}=\mathbf{1}^{\Lambda\left(T^{*} M\right)} \otimes R^{\mathcal{V}}
$$

into Equation (139), and decomposing a section $\psi$ of the twisted vector bundle as

$$
\psi=\sum_{i, j} a_{i, j} c_{i} \otimes f_{j}
$$


where $\left\{f_{j}\right\}_{j}$ an o.n.b of $L^{2}\left(\Omega, \mathcal{V}, \mathcal{A}_{0}, d P\right)$, and $\left\{c_{i}\right\}_{i}$ is an o.n.b of $L^{2}\left(\Omega, \Lambda\left(T^{*} M\right), \mathcal{A}_{0}, d P\right)$ of eigenvectors of $\gamma^{\Lambda\left(T^{*} M\right)}\left(e_{\mu}\right)$ such that for all $\mu$,

$$
\gamma^{\Lambda\left(T^{*} M\right)}\left(e_{\mu}\right) c_{i}=(-1)^{i} \iota
$$

we obtain

$$
\begin{aligned}
\left(\mathcal{R}^{W} \psi, \psi\right) & =-\sum_{\substack{i, j, k, l \\
\mu \neq v}} a_{i, j} a_{k, l}(-1)^{\mu+v} \delta_{i, k}\left(R^{\mathcal{V}}\left(e_{\mu}, e_{v}\right) f_{j}, f_{l}\right) \\
& =\sum_{\substack{i, j, k, l \\
v \neq \mu}} a_{i, j} a_{k, l}(-1)^{v+\mu} \delta_{i, k}\left(R^{\mathcal{V}}\left(e_{\nu}, e_{\mu}\right) f_{j}, f_{l}\right) \\
& =-\left(\mathcal{R}^{W} \psi, \psi\right),
\end{aligned}
$$

and hence, for any $\psi$

$$
\left(\mathcal{R}^{W} \psi, \psi\right)=0 .
$$

Based on the polarization identity, we infer $\mathcal{R}^{W}=0$, and Equation (137) follows. For the proof of (138), we have:

$\Rightarrow$ If NFLVR holds, then with Theorem 11 there exists an $f \in \operatorname{dom}\left(\Delta_{B_{N}}^{\mathcal{V}}\right)$ such that $\Delta^{\mathcal{V}} f=0$, and $\nabla^{\mathcal{V}}=0$. Let us consider the section $\psi:=c \otimes f$, where $c \in \mathbb{R}$ is a constant. With Equation (137), we infer via integration by parts

$$
\left(Q^{W^{2}} \psi, \psi\right)=\left(\nabla^{W^{*}} \nabla^{W} \psi, \psi\right)=\left(\nabla^{W} \psi, \nabla^{W} \psi\right)=c^{2}\left(\nabla^{\mathcal{V}} f, \nabla^{\mathcal{V}} f\right)=0,
$$

and the proof in this direction is complete.

$\Leftarrow:$ If there is a $\psi \neq 0$ in $\left.\operatorname{ker}\left(Q_{B_{\text {abs }}^{0}}^{W} \otimes B_{N}\right)^{2}\right)$, then

$$
\psi=\bigoplus_{i, j} a_{i, j} c_{i} \otimes f_{j}
$$

where $c_{i} \in \Lambda^{i}\left(T^{*} M\right)$, because the Laplace-Beltrami operator maintains the degree of differential forms. Therefore, there is at least a pair $(i, j)$ such that for $c_{i} \neq 0$ and $f_{j} \neq 0$

$$
Q^{W^{2}} c_{i} \otimes f_{j}=0,
$$

and based on Equation (137),

$$
\begin{aligned}
0 & =\left(Q^{W^{2}} c_{i} \otimes f_{j}, c_{i} \otimes f_{j}\right)=\left(\nabla^{W}\left(c_{i} \otimes f_{j}\right), \nabla^{W}\left(c_{i} \otimes f_{j}\right)\right) \\
& =\left(\nabla^{\Lambda^{i}\left(T^{*} M\right)} c_{i}, \nabla^{\Lambda^{i}\left(T^{*} M\right)} c_{i}\right)\left(\nabla^{\mathcal{V}} f_{j}, \nabla^{\mathcal{V}} f_{j}\right),
\end{aligned}
$$

and therefore, $\nabla^{\mathcal{V}} f_{j}=0$ for at least a $j$. Hence, $\Delta^{\mathcal{V}} f_{j}=0$, and NFLVR follows from Theorem 11.

Remark 22. Theorem 16 holds true if we replace the absolute with the relative boundary condition.

Corollary 4. The no-free-lunch-with-vanishing-risk condition is satisfied if and only if $H_{*}(M, \mathcal{V})$, the homology group of the vector bundle $V$ over $M$, does not vanish:

$$
(N F L V R) \Longleftrightarrow H_{*}(M, \mathcal{V}) \neq\{0\} .
$$


Proof. According to Theorem 16, NFLVR and the isomorphism between the cohomology group and the kernel of $\left(Q_{B_{\mathrm{abs}}^{0}}^{W} \otimes B_{N}\right)^{2}$

$$
H^{*}\left(M, \Lambda\left(T^{*} M\right) \otimes \mathcal{V}\right)=\bigoplus_{j=0}^{N} H\left(M, \Lambda^{j}\left(T^{*} M\right) \otimes H^{*}(M, \mathcal{V})=\bigoplus_{j=0}^{N} H^{j}(M) \otimes H^{*}(M, \mathcal{V})\right.
$$

where $H^{j}(M, \mathbb{R})$ is the $j$ th cohomology group of $M$ and $H^{*}(M, \mathcal{V})$ the cohomology group of the vector bundle $V$ over $M$. We see that NFLVR is satisfied if and only if there exists at least a $j=0, \ldots, N+1$ such that

$$
H^{j}(M, \mathbb{R}) \otimes H^{*}(M, \mathcal{V}),
$$

Since $H^{0}(M) \neq\{0\}$ because the constant functions are eigenvectors of the LaplaceBeltrami operator under the absolute boundary condition for the eigenvalue 0 , this condition is satisfied if and only if the cohomology group of the cash flow bundle does not vanish. Since the cohomology group is isomorphic to the homology group, the proof is complete.

Remark 23. Corollary 4 states that the vanishing of the homology group of the cash flow bundle is a topological obstruction to the no-free-lunch-with-vanishing-risk condition.

\section{Conclusions}

By introducing an appropriate stochastic differential geometric formalism, the classical theory of stochastic finance can be embedded into a conceptual framework called Geometric Arbitrage Theory, where the market is modelled with a principal fibre bundle with a connection and arbitrage corresponding to its curvature. The associated vector bundle, termed cash flow bundle, carries a covariant differentiation induced by the connection. The presence of the eigenvalue 0 in the spectrum of the connection Laplacian on the cash flow bundle, or of the Dirac Laplacian on the cash flow bundle twisted with the exterior algebra bundle, characterizes the fulfilment of the no-free-lunch-with-vanishing-risk condition for the market model. We extend the Jarrow-Protter-Shimbo bubble theory to markets allowing for arbitrage and highlight the connections with the Platen-Heath real world pricing of the benchmark approach. The non-vanishing of the Euler characteristic of the asset nominal space and the non-vanishing of the homology group of the cash flow bundle are topological obstructions to the fulfillment of the NFLVR condition.

As a result, we have justified the title of this paper, which is an adaptation of Kač's famous question that introduced spectral inverse problems for self-adjoint operators on manifolds: Can you hear the shape of a drum? We do hope that this approach will be appreciated by the mathematical finance community.

Author Contributions: Both authors contributed in equal manner to the conceptualization, the methodology and the validation of this publication. All authors have read and agreed to the published version of the manuscript.

Funding: This research received no external funding.

Institutional Review Board Statement: Not applicable.

Informed Consent Statement: Not applicable.

Data Availability Statement: No data were utilized.

Conflicts of Interest: The authors declare no conflict of interest.

\section{Appendix A. Generalized Derivatives of Stochastic Processes}

In stochastic differential geometry, one would like to lift the constructions of stochastic analysis from open subsets of $\mathbf{R}^{N}$ to $N$ dimensional differentiable manifolds. To that 
aim, chart invariant definitions are needed, and hence, a stochastic calculus satisfying the usual chain rule instead of Itô's Lemma is required, (cf. [14], Chapter 7, and the remark in Chapter 4 at the beginning of page 200). That is why the papers about geometric arbitrage theory are mainly concerned with stochastic integrals and derivatives meant in Stratonovich's sense and not in Itô's. Of course, at the end of the computation, Stratonovich integrals can be transformed into Itô's. Note that a fundamental portfolio equation, the selffinancing condition, cannot be directly formally expressed with Stratonovich integrals; it must first be expressed with Itô's and then transformed into Stratonovich's because it is a non-anticipative condition.

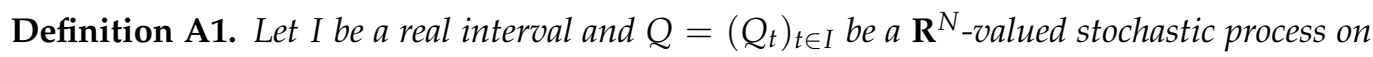
the probability space $(\Omega, \mathcal{A}, P)$. The process $Q$ determines three families of $\sigma$-subalgebras of the $\sigma$-algebra $\mathcal{A}$ :

(i) "Past" $\mathcal{P}_{t}$, generated by the preimages of Borel sets in $\mathbf{R}^{N}$ by all mappings $Q_{s}: \Omega \rightarrow \mathbf{R}^{N}$ for $0<s<t$

(ii) "Future" $\mathcal{F}_{t}$, generated by the preimages of Borel sets in $\mathbf{R}^{N}$ by all mappings $Q_{s}: \Omega \rightarrow \mathbf{R}^{N}$ for $0<t<s$;

(iii) "Present" $\mathcal{N}_{t}$, generated by the preimages of Borel sets in $\mathbf{R}^{N}$ by the mapping $Q_{s}: \Omega \rightarrow \mathbf{R}^{N}$. Let $Q=\left(Q_{t}\right)_{t \in I}$ be continuous. Assuming that the following limits exist, Nelson's stochastic derivatives are defined as follows:

$$
\begin{aligned}
& \mathfrak{D} Q_{t}:=\lim _{h \rightarrow 0^{+}} \mathbb{E}\left[\frac{Q_{t+h}-Q_{t}}{h} \mid \mathcal{P}_{t}\right]: \text { forward derivative, } \\
& \mathfrak{D}_{*} Q_{t}:=\lim _{h \rightarrow 0^{+}} \mathbb{E}\left[\frac{Q_{t}-Q_{t-h}}{h} \mid \mathcal{F}_{t}\right]: \text { backward derivative, } \\
& \mathcal{D} Q_{t}:=\frac{\mathfrak{D} Q_{t}+\mathfrak{D}_{*} Q_{t}}{2}: \text { mean derivative. }
\end{aligned}
$$

Let $\mathcal{S}^{1}(I)$ be the set of all processes $Q$ such that $t \mapsto Q_{t}, t \mapsto \mathfrak{D} Q_{t}$ and $t \mapsto \mathfrak{D}_{*} Q_{t}$ are continuous mappings from $I$ to $L^{2}(\Omega, \mathcal{A})$. Let $\mathcal{C}^{1}(I)$ be the completion of $\mathcal{S}^{1}(I)$ with respect to the norm

$$
\|Q\|:=\sup _{t \in I}\left(\left\|Q_{t}\right\|_{L^{2}(\Omega, \mathcal{A})}+\left\|\mathfrak{D} Q_{t}\right\|_{L^{2}(\Omega, \mathcal{A})}+\left\|\mathfrak{D}_{*} Q_{t}\right\|_{L^{2}(\Omega, \mathcal{A})}\right)
$$

Remark A1. The stochastic derivatives $\mathfrak{D}, \mathfrak{D}_{*}$, and $\mathcal{D}$ correspond to Itô's, to the anticipative, and, respectively, to Stratonovich's integral (cf. [33]). The process space $\mathcal{C}^{1}(I)$ contains all of Itô processes. If $Q$ is a Markov process, then the sigma algebras $\mathcal{P}_{t}$ ("past") and $\mathcal{F}_{t}$ ("future") in the definitions of forward and backward derivatives can be substituted by the sigma algebra $\mathcal{N}_{t}$ ("present") (see Chapter 6.1 and 8.1 in [33]).

Stochastic derivatives can be defined pointwise in $\omega \in \Omega$ outside the class $\mathcal{C}^{1}$ in terms of generalized functions.

Definition A2. Let $Q: I \times \Omega \rightarrow \mathbf{R}^{N}$ be a continuous linear functional in the test processes $\varphi: I \times \Omega \rightarrow \mathbf{R}^{N}$ for $\varphi(\cdot, \omega) \in C_{c}^{\infty}\left(I, \mathbb{R}^{N}\right)$. This means that for a fixed $\omega \in \Omega$, the functional $Q(\cdot, \omega) \in \mathcal{D}\left(I, \mathbb{R}^{N}\right)$, the topological vector space of continuous distributions. We can then define Nelson's generalized stochastic derivatives:

$$
\begin{aligned}
& \mathfrak{D} Q\left(\varphi_{t}\right):=-Q\left(\mathfrak{D} \varphi_{t}\right): \text { forward generalized derivative; } \\
& \mathfrak{D}_{*} Q\left(\varphi_{t}\right):=-Q\left(\mathfrak{D}_{*} \varphi_{t}\right): \text { backward generalized derivative; } \\
& \mathcal{D}\left(\varphi_{t}\right):=-Q\left(\mathcal{D} \varphi_{t}\right): \text { mean generalized derivative. }
\end{aligned}
$$


If the generalized derivative is regular, then the process has a derivative in the classic sense. This construction is nothing other than a straightforward pathwise lift of the theory of generalized functions to a wider class of stochastic processes which do not a priori allow for Nelson's derivatives in the strong sense. We will utilize this feature in the treatment of credit risk, where many processes with jumps occur.

\section{References}

1. Malaney, P.N. The Index Number Problem: A Differential Geometric Approach. Ph.D. Thesis, Harvard University Economics Department, Cambridge, MA, USA, 1996.

2. Weinstein, E. Gauge Theory and Inflation: Enlarging the Wu-Yang Dictionary to a unifying Rosetta Stone for Geometry in Application; Talk Given at Perimeter Institute: : Waterloo, ON, Canada; Perimeter Institute: Waterloo, ON, Canada, 2006.

3. Ilinski, K. Gauge Geometry of Financial Markets. J. Phys. A Math. Gen. 2000, 33, L5-L14. [CrossRef]

4. Ilinski, K. Physics of Finance: Gauge Modelling in Non-Equilibrium Pricing; Wiley: Hoboken, NJ, USA, 2001.

5. Young, K. Foreign Exchange Market as a Lattice Gauge Theory. Am. J. Phys. 1999, 67, 862-868. [CrossRef]

6. Smith, A.; Speed, C. Gauge Transforms in Stochastic Investment. In Proceedings of the 1998 AFIR Colloquim, Cambridge, UK, 14-17 September 1998.

7. Flesaker, B.; Hughston, L. Positive Interest. Risk 1996, 9, 46-49.

8. Hugonnier, J.; Prieto, R. Asset Pricing with Arbitrage Activity. J. Financ. Econ. 2015, 115, 411-428. [CrossRef]

9. Ruf, J. Hedging under Arbitrage. Math. Financ. 2013, 23, 297-317.

10. Farinelli, S. Geometric Arbitrage Theory and Market Dynamics. J. Geom. Mech. 2015, 7, 431-471. [CrossRef]

11. Farinelli, S.; Takada, H. The Black-Scholes Equation in Presence of Arbitrage. 2021, submitted preprint. [CrossRef]

12. Elworthy, K.D. Stochastic Differential Equations on Manifolds; London Mathematical Society Lecture Notes Series; Cambridge University Press: Cambridge, UK, 1982.

13. Eméry, M. Stochastic Calculus on Manifolds-With an Appendix by P. A. Meyer; Springer: Berlin/Heidelberg, Germany, 1989.

14. Hackenbroch, W.; Thalmaier, A. Stochastische Analysis. Eine Einführung in die Theorie der Stetigen Semimartingale; Teubner Verlag: Stuttgart, Germany, 1994.

15. Hsu, E.P. Stochastic Analysis on Manifolds; Graduate Studies in Mathematics; AMS: Providence, RI, USA, 2002; Volume 38.

16. Schwartz, L. Semi-Martingales sur des Variétés et Martingales Conformes sur des Variétés Analytiques Complexes; Springer Lecture Notes in Mathematics; Springer: Berlin/Heidelberg, Germany, 1980.

17. Stroock, D.W. An Introduction to the Analysis of Paths on a Riemannian Manifold; Mathematical Surveys and Monographs; AMS: Providence, RI, USA, 2000; Volume 74.

18. Hunt, P.J.; Kennedy, J.E. Financial Derivatives in Theory and Practice; Wiley Series in Probability and Statistics; John Wiley and Sons: Hoboken, NJ, USA, 2004.

19. Delbaen, F.; Schachermayer, W. The Mathematics of Arbitrage; Springer: Berlin/Heidelberg, Germany, 2008.

20. Delbaen, F.; Schachermayer, W. A General Version of the Fundamental Theorem of Asset Pricing. In Mathematische Annalen; Springer: Berlin/Heidelberg, Germany, 1994; Volume 300, pp. 463-520.

21. Kabanov, Y.M. On the FTAP of Kreps-Delbaen-Schachermayer; Statistics and control of stochastic processes; World Scientific Publishing Company: Moscow, Russia, 1997; pp. 191-203.

22. Jarrow, R.; Larsson, M. The Meaning of Market Efficiency. Math. Financ. 2012, 22, 1-30. [CrossRef]

23. Jarrow, R. Third Fundam. Theorem Asset Pricing. Annals Financ. Econ. 2012, 2, 1-11.

24. Hörmander, L. The Analysis of Linear Partial Differential Operators I: Distribution Theory and Fourier Analysis; Springer: Berlin/Heidelberg, Germany, 2003.

25. Bleecker, D. Gauge Theory and Variational Principles; Republished by Dover 2005; Addison-Wesley Publishin: Boston, MA, USA, 1981.

26. Baum, H. Eichfeldtheorie: Eine Einführung in die Differentialgeometrie auf Faserbündeln; Springer Spektrum: Champaign, IL, USA, 2014.

27. Jarrow, R.; Protter, P.; Shimbo, K. Asset Price Bubbles in Incomplete Markets. Math. Financ. 2010, 20, 145-185. [CrossRef]

28. Heath, D.; Platen, E. A Benchmark Approach to Quantitative Finance; Springer: Berlin/Heidelberg, Germany, 2006.

29. Protter, P. A Mathematical Theory of Financial Bubbles. In Paris-Princeton Lectures on Mathematical Finance; Springer Lecture Notes in Mathematics 2081; Springer: Berlin/Heidelberg, Germany, 2013.

30. Gilkey, P.B. Invariance Theory, the Heat Equation and the Atiyah-Singer Index Theorem, 2nd ed.; Studies in Advanced Mathematics; CRC Press: Boca Raton, FL, USA, 1995.

31. Berline, N.; Getzler, E.; Vergne, M. Heat Kernels and Dirac Operators; Corrected Second Printing, Grundlehren der Mathematischen Wissenschaften; Springer: Berlin/Heidelberg, Germany, 1996.

32. Lawson, H.B.; Michelson, M.-L. Spin Geometry; Princeton Mathematical Series; Princeton University Press: Princeton, NJ, USA, 1989; Volume 38.

33. Gliklikh, Y.E. Global and Stochastic Analysis with Applications to Mathematical Physics; Theoretical and Mathemtical Physics; Springer: Berlin/Heidelberg, Germany, 2010. 